\title{
Stacking-fault mediated plasticity and strengthening in lean, rare- earth free magnesium alloys
}

\section{Journal Article}

\section{Author(s):}

Basu, Indranil (D); Chen, Ming; Wheeler, Jeffrey (D); Schäublin, Robin E.; Löffler, Jörg F.

Publication date:

2021-06-01

Permanent link:

https://doi.org/10.3929/ethz-b-000479922

Rights / license:

Creative Commons Attribution-NonCommercial-NoDerivatives 4.0 International

Originally published in:

Acta Materialia 211, https://doi.org/10.1016/j.actamat.2021.116877

Funding acknowledgement:

172934 - Advanced nanoscale characterization of magnetic defects in metals (SNF) 
Full length article

\title{
Stacking-fault mediated plasticity and strengthening in lean, rare-earth free magnesium alloys
}

\author{
I. Basu ${ }^{\mathrm{a}, *}$, M. Chen ${ }^{\mathrm{b}}$, J. Wheeler ${ }^{\mathrm{b}}$, R.E. Schäublin ${ }^{\mathrm{a}}$, J.F. Löffler ${ }^{\mathrm{a}, *}$ \\ a Laboratory of Metal Physics and Technology, Department of Materials, ETH Zurich, 8093Zurich, Switzerland \\ ${ }^{\mathrm{b}}$ Laboratory for Nanometallurgy, Department of Materials, ETH Zurich, 8093Zurich, Switzerland
}

\section{A R T I C L E I N F O}

\section{Article history:}

Received 17 December 2020

Revised 12 March 2021

Accepted 3 April 2021

Available online 7 April 2021

\section{Keywords:}

Magnesium

Twin nucleation

dislocation analysis

Chemical ordering

Stacking-fault energy

\begin{abstract}
A B S T R A C T
A magnesium alloy with lean additions of $\mathrm{Zn}(1 \mathrm{wt} . \%)$ and $\mathrm{Ca}(0.3 \mathrm{wt} . \%), \mathrm{ZX} 10$, and pure Mg were subjected to orientation-dependent micropillar indentation tests at ambient temperature. Single-crystalline micropillars of two different orientations were fabricated to activate extension and compression along the $c$-axis, respectively. For both loading conditions, ZX10 exhibits a strengthening increment by a factor of 2 to 2.5 compared to pure $\mathrm{Mg}$ along with plasticity enhancement. Correlative transmission electron backscattered diffraction and transmission electron microscopy reveal that deformation in ZX10 proceeds by deformation twinning under c-axis extension, generating homogeneous activation of basal and non-basal slip at higher strains. In contrast, pure Mg displays deformation through tension twinning and basal slip. Pure Mg under c-axis compression deforms by basal dislocation-mediated massive sliding, while ZX10 reveals dual activation of basal $\langle a\rangle$ and pyramidal $\langle c+a\rangle$ dislocations. Mechanistically, the minute additions of $\mathrm{Zn}$ and $\mathrm{Ca}$ solutes modify the intrinsic stacking-fault energy, which accounts for the simultaneous strengthening and ductility enhancement. These findings highlight the beneficial impact of dilute additions of $\mathrm{Zn}$ and $\mathrm{Ca}$ in activating novel deformation pathways that are critical for designing rare-earth (RE) free high-strength, highly ductile magnesium alloys for structural and biomedical applications.
\end{abstract}

(C) 2021 The Author(s). Published by Elsevier Ltd on behalf of Acta Materialia Inc. This is an open access article under the CC BY-NC-ND license (http://creativecommons.org/licenses/by-nc-nd/4.0/)

\section{Introduction}

The past decade has witnessed a renewed interest in lightweight magnesium $(\mathrm{Mg})$ alloys, stimulated by urgent weightsaving and thereby energy-saving requirements in automotive, aerospace and communication industries [1-6]. Despite the tremendous underlying potential for $\mathrm{Mg}$ alloys to emerge as nextgeneration structural materials, their widespread use is considerably limited owing to the overarching problem of poor strengthductility synergy [7]. Present magnesium alloys show limited cold formability due to their restricted plasticity at ambient temperatures, primarily stemming from: (i) the absence of five or more independent deformation modes required to fulfil the von-Mises criterion for homogeneous plastic deformation, and (ii) the generation of strong crystallographic textures during deformation that promote mechanical anisotropy [2,8-11]. Both outcomes are fundamentally attributed to the low crystal symmetry in an hexagonal

\footnotetext{
* Corresponding authors.

E-mail addresses: indranil.basu@mat.ethz.ch (I. Basu), joerg.loeffler@mat.ethz.ch (J.F. Löffler).
}

close-packed (hcp) structure, wherein strain accommodation along the $a$-axis (i.e. on (0002) basal planes) is strongly preferred while slip deformation along the $c$-axis (i.e. on non-basal planes) is difficult to activate $[2,8]$.

Numerous studies have indicated that through intelligent alloying and appropriate thermomechanical treatment the activation and stability of dislocations and slip systems can be manipulated, such that $\mathrm{Mg}$ alloys can display intrinsic improvements in their room-temperature plasticity response [10,12-15]. This has in particular been established for $\mathrm{Mg}$ alloys with dilute additions of rareearth elements (REEs), where their presence promotes the relative activity of non-basal slip, i.e. pyramidal $\langle c+a\rangle$ dislocations, which provide an out-of-basal plane shear [3,12,16,17]. Moreover, activation of pyramidal slip provides five independent deformation modes, giving rise to homogeneous plasticity and strain-hardening response [8]. Despite the advantages of $\mathrm{Mg}-\mathrm{RE}$-based alloys, their commercial applications are rather limited, owing to an expensive and cumbersome processing scheme plus poor recyclability $[18,19]$. In order to mitigate the aforementioned issues, the Mg-research community has lately shown great interest in the search of alternative low-cost REE-free Mg alloys. This has motivated the develop- 
ment of new classes of $\mathrm{Mg}$ alloys, particularly those containing $\mathrm{Ca}$, which because of their excellent bioresorbability and low cytotoxicity are also suitable candidates for deployment as biodegradable medical implants [19-22].

In recent years, $\mathrm{Mg}-\mathrm{Ca}$-based alloys have shown great promise as structural materials, displaying easy formability and high agehardenability $[19,21,23,24]$. In a seminal work [12], atomistic simulations were utilized to correlate the impact of alloying elements on the stacking-fault energy and intrinsic ductility of $\mathrm{Mg}$ alloys. It was predicted that both RE and Ca solutes would effectively lower the intrinsic stacking-fault energy, which is a key contributor for enhancing material ductility. Furthermore, in the presence of lean additions of $\mathrm{Zn}$, this effect is expected to be further enhanced [3,12]. However, unlike solid-solution Mg-RE-based alloys, where an extended repository of experimental studies on the effect of RE solutes on the overall mechanical response in $\mathrm{Mg}$ alloys is available $[2,3,10,11,13-15,25-32]$, our understanding of solid-solution Mg-Ca-based alloys and its impact on intrinsic plasticity mechanisms is rather incomplete. In studies of Ca-containing $\mathrm{Mg}$ alloys that date back more than two decades, the primary focus was and still is pinned upon the precipitation-hardening response in these alloys $[19,33,34]$.

The present work attempts to unravel the key plasticity mechanisms in Mg-Zn-Ca alloys with special emphasis on the role of solute $\mathrm{Ca}$ and $\mathrm{Zn}$ atoms in modifying the deformation response. Pure $\mathrm{Mg}$ is used as a reference material in this study. A combination of microcompression testing and correlative electron microscopy is implemented to probe the structure-property relationships. In particular, definite correlations between solute arrangement in the $\mathrm{Mg}$ matrix and its resultant influence on the spatial distribution of defects are established. Moreover, the influence of alloying elements on the slip and twinning deformation is assessed both qualitatively and quantitatively.

\section{Experimental methods}

As-extruded Mg-1wt.\%Zn-0.3wt.\%Ca (ZX10) alloys (more details in Ref. [24]) were solutionized at $520{ }^{\circ} \mathrm{C}$ for 2 hrs to dissolve all $\mathrm{Zn}$ and $\mathrm{Ca}$ in the $\mathrm{Mg}$ matrix. The specimens were then cut along the transverse direction such that the viewing plane was parallel to the extrusion direction. For the purpose of comparison, specimens were also cut from as-cast, homogenized commercially-pure Mg. To obtain the size distribution and orientation of the grains within the microstructure, conventional electron back-scattered diffraction (EBSD) measurements were performed using an FEI Quanta 200 field-emission gun scanning electron microscope (SEM) equipped with an EDAX ${ }^{\mathrm{TM}}$ Hikari EBSD camera. The specimens for EBSD on both ZX10 and pure Mg were prepared using conventional metallography techniques, with $\mathrm{Ar}^{+}$broad ion-beam milling (Hitachi IM4000) as a final step. The broad ion-beam milling was performed in two steps, where an initial short-duration ion-beam etching removed the top damaged layer resulting from preparatory grinding, and a subsequent long-duration polishing was performed at low accelerating voltage $(2 \mathrm{kV})$ under a grazing-incidence angle.

Two grain orientations, i.e. $\langle 10 \overline{1} 0\rangle \| \mathrm{CD}$ and $\langle 0002\rangle \| \mathrm{CD}$ (where $\mathrm{CD}$ denotes the compression direction; TD is the transverse direction), were selected for both pure $\mathrm{Mg}$ and ZX10 to fabricate specially oriented single-crystalline micro-pillars, using a $\mathrm{Ga}^{+}$focused ion-beam (Helios Nanolab 600i) operated at an accelerating voltage of $30 \mathrm{kV}$. For each condition, i.e. orientation and material type, at least six individual pillars with diameters of $2 \mu \mathrm{m}$ and heights of $5 \mu \mathrm{m}$ were milled. Three different milling currents were employed: $9.4 \mathrm{nA}$ for coarse milling, followed by a medium current of $0.77 \mathrm{nA}$ to dimension the pillar close to the designed geometry, and a final surface polishing with $33 \mathrm{pA}$ to minimize the taper, such that a resultant taper angle of $<1^{\circ}$ was achieved.
The micro-compression testing of the milled pillars was performed in situ inside an SEM (Tescan Vega 3, Czech Republic), using an instrumented indentation system (Alemnis AG, Switzerland) under displacement-control mode at room temperature and deploying a diamond flat-punch (Synton-MDP, Switzerland). All pillars were compressed at a constant strain rate of $1 \times 10^{-2} \mathrm{~s}^{-1}$. The chosen pillar orientations under uniaxial compression resulted in crytallographically distinct loading modes with c-axis extension in one scenario ( $\langle 10 \overline{1} 0\rangle \| \mathrm{CD})$ and c-axis compression ( $\langle 0002\rangle \| \mathrm{CD})$ in the other. The engineering stress-strain curves were calculated from the measured load-displacement data and the initial top surface area of the pillars. Three pillars corresponding to each condition (orientation and material type) were deformed until failure to obtain a statistically relevant estimate of the final deformation strain. The averaged data over these 3 independent tests is presented as representative mechanical data for each orientation. The yield stress was determined as the stress value corresponding to the first excursion from the linear stress-strain response. The critical resolved shear-stresses corresponding to the active deformation mode were quantified by multiplying the measured yield stress with the Schmid factor of the active slip system. In order to evaluate the plasticity mechanisms as a function of strain, additional micro-pillar compression tests were stopped at intermediate plastic strain values.

Nanoscale structural characterization of the micropillars compressed to selected strains was performed using correlative transmission EBSD (t-EBSD) and transmission electron microscopy (TEM). Cross-sections of deformed micropillars were prepared using standard lift-out techniques in the FIB using a micromanipulator. The cross-sections were thinned down to electron transparency with graded reduction of milling voltage and current, with the final polishing step performed at $2 \mathrm{kV}$ for a milling current of $10 \mathrm{pA}$. t-EBSD measurements were performed in the FEI Quanta 200 FEG-SEM at an accelerating voltage of $30 \mathrm{kV}$ and a beam spot size of $4 \mathrm{~nm}$. The specimen was tilted at an angle of $-5^{\circ}$, and a step size of $10 \mathrm{~nm}$ and a hexagonal grid type was implemented. A binning of $4 \times 4$ was used when capturing the Kikuchi patterns, producing a $320 \times 240$ CCD camera resolution. The acquired raw EBSD data was subsequently analyzed using EDAX-TSL OIM ${ }^{\mathrm{TM}}$ Analysis 8 software and an MTEX Matlab toolbox [35]. In order to achieve high indexing rates, the raw unfiltered EBSD data was reindexed employing a neighborhood-pattern averaging (n-PAR) scheme to improve the pattern's signal-to-noise ratio [36]. The n-PAR algorithm is available as a built-in function in the conventional EBSD analysis software OIM $^{\mathrm{TM}}$ Analysis 8. Noise filtering was performed with a threshold confidence interval of 0.2 .

TEM imaging of the micropillar cross-sections was performed in a JEOL JEM-1400 transmission electron microscope operated at $120 \mathrm{kV}$ using a JEOL double-tilt sample holder. Elemental distribution maps were recorded by electron dispersive X-ray spectroscopy (EDS) measurements that were conducted using an FEI Talos 200X transmission electron microscope, operated at $200 \mathrm{kV}$, with an inbuilt FEI Super $\mathrm{X}$ detector equipped with large-collection angles technology.

In order to identify the active dislocation and stacking-fault mechanisms, a quantitative interpretation of TEM micrographs was accomplished by means of TEM image simulations of the observed straight dislocation lines and stacking faults in $\mathrm{Mg}$. We considered one dislocation bordering one stacking fault. Simulations were performed using CUFOUR software, which combines the manybeam Schäublin-Stadelmann equations to describe the propagation of the electron beam in the TEM thin foil and anisotropic elasticity to describe the deformation of the material around defects [37]. The general computational sequence in CUFOUR consists of three main parts: (i) preprocessing of geometrical and physical parameters input to describe the sample conditions (ma- 


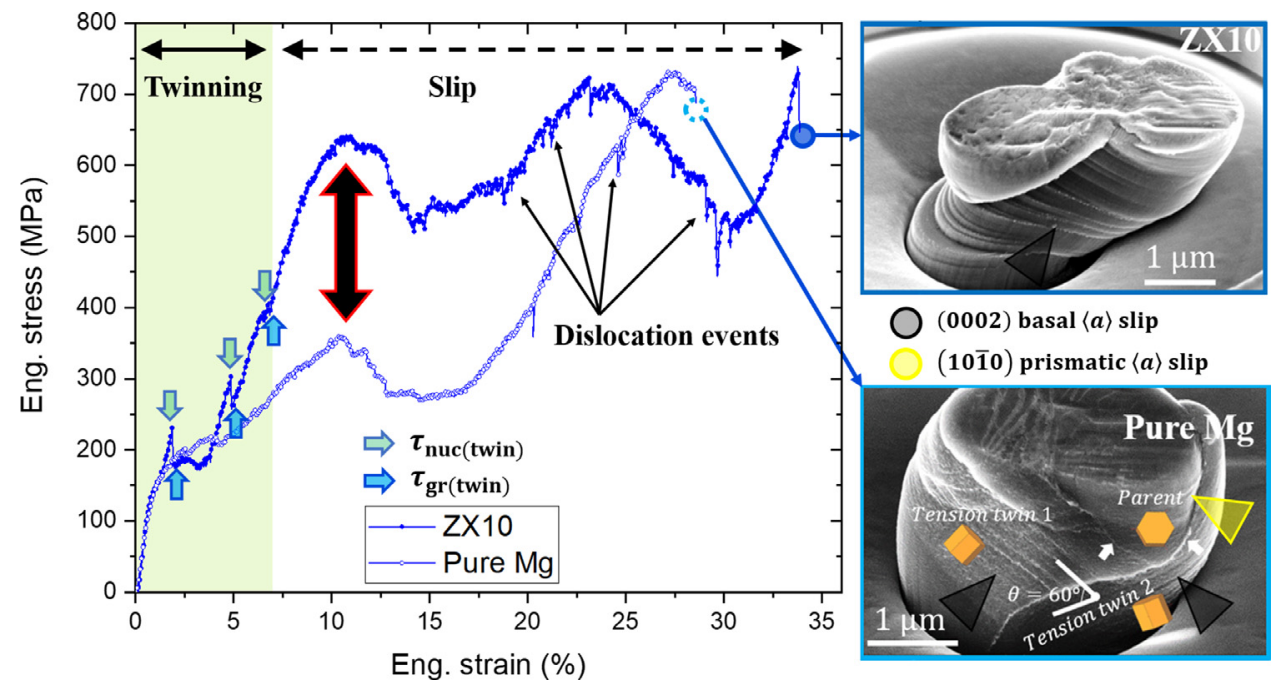

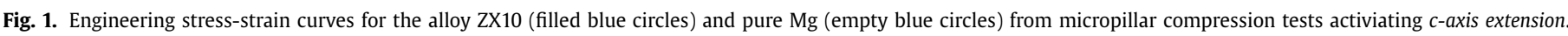

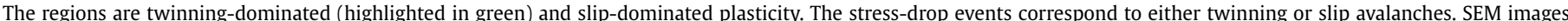

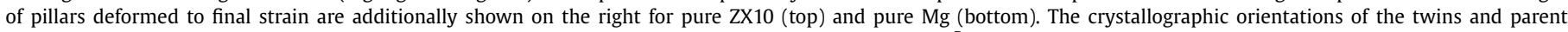
grains are depicted for pure Mg; the basal- $\langle a\rangle$ slip traces are revealed by black arrow heads and the $\{10 \overline{1} 0\}$ prismatic- $\langle a\rangle$ slip traces are shown by yellow ones.

terial type, lamella condition) containing the defects of interest; (ii) solving Schäublin-Stadelmann equations; (iii) postprocessing of the simulated TEM image. The physical parameters for describing the orientation of the TEM lamella inside the microscope were carefully chosen. The electron beam direction, the foil normal and the diffraction condition, which includes the operating diffraction vector and its excitation, were matched with the experiments according to the electron diffraction patterns. The corresponding defect configuration made up of one dislocation bording one stacking fault was chosen among all those that are found in hexagonal metals including $\mathrm{Mg}$. This includes: the dislocation Burgers vector, the dislocation line, the stacking-fault habit plane and the lattice translation across the stacking fault. The simulated image that best matches the experimental image then reveals the exact dislocation-stacking-fault configuration. The thickness of the foil was established by matching the simulated and experimental images. The considered elastic constants for $\mathrm{Mg}$ [38] were: $C_{11}=C_{22}=59.4 \mathrm{GPa}, C_{12}=25.6 \mathrm{GPa}, C_{13}=21.4 \mathrm{GPa}$, $C_{33}=61.6 \mathrm{GPa}, C_{44}=C_{55}=16.4 \mathrm{GPa}, C_{66}=16.9 \mathrm{GPa}$.

\section{Results}

\subsection{Stress-strain response under micropillar compression}

Fig. 1 shows the engineering stress-strain response of ZX10 under $c$-axis extension in comparison to the mechanical response of pure $\mathrm{Mg}$ under the same loading conditions. The initial stressstrain responses for both ZX10 and pure Mg are similar, with the onset of plasticity in both cases occurring at a strain of $\varepsilon \approx$ $0.75 \%$ (with a corresponding stress of $\sim 120 \mathrm{MPa}$ ). As the imposed strain becomes larger than $\varepsilon \approx 1.5 \%$, the hardening response of ZX10 markedly increases (highlighted by a black double-edged arrow in Fig. 1) and deviates from the trend exhibited by pure $\mathrm{Mg}$, culminating into a sharp load drop at $\varepsilon \approx 2 \%$. This is succeeded by a short stress plateau, which typically indicates the onset of twinning activity and has been well established in earlier studies $[39,40]$. Such characteristically similar stress spikes occur multiple times up to a strain of $\varepsilon \approx 7 \%$. Fig. 1 highlights the twinnucleation and twin-growth events by green and blue arrows, respectively. At higher strains, the stress-strain curve shows multiple hardening regimes interspersed with softening, highlighted by a series of stress drop events. Loading under c-axis extension for pure Mg exhibits qualitatively similar behavior as seen for ZX10, corresponding to twinning-dominated deformation up to a strain of $\varepsilon \approx 7 \%$. However, the hardening rate is significantly lower compared to ZX10. The stress-strain response at higher strains is highlighted by alternating hardening and softening behavior until failure at $\varepsilon \approx 29 \%$.

SEM micrographs of the micropillars deformed to final strain are shown on the right-hand side of Fig. 1. The bottom right image represents the specimen geometry corresponding to the deformation of pure $\mathrm{Mg}$ under c-axis extension. Geometrically, the postdeformation pillar aspect ratio is significantly modified, as indicated by a reduced height and a slight expansion along the lateral dimension. Deformation is dominated by twinning, giving rise to two tension twin variants as shown by the $60^{\circ}$ misorientation between the basal planes of adjacent twins. The top part of the pillar retains the parent orientation, as revealed by the twin boundaries illustrated by white arrows. In terms of dislocation slip, the parent grain shows slip traces corresponding to prismatic $\langle a\rangle$ slip activity. Both twins undergo profuse basal slip, illustrated by the high density of basal slip traces. In contrast, the micropillar geometry of the ZX10 alloy (shown in the top right corner of Fig. 1) appears to retain its initial aspect ratio with relatively less defined expansion along the lateral dimension. However, the pillar displays a bent geometry owing to a series of visibly discrete basal dislocation-slip shearing events, generating successive slip steps in the direction lateral to the pillar.

Fig. 2 shows micropillar compression tests for ZX10 and pure $\mathrm{Mg}$, corresponding to c-axis compression. A loading parallel to the $\langle 0002\rangle$ direction clearly indicates higher elastic stiffness in the case of pure $\mathrm{Mg}$ compared to ZX10. While the former reveals a yield stress of $387 \mathrm{MPa}$, the latter shows a yield stress value of $323 \mathrm{MPa}$. The strain increment is characterized by a higher hardening slope for ZX10 compared to pure Mg up to a strain of $\varepsilon \approx 5 \%$, where both reach a stress value of $600 \mathrm{MPa}$. At higher strains, pure $\mathrm{Mg}$ displays massive softening, where the stress drops to 300 MPa. In contrast, the ZX10 alloy continues to show strain hardening with intermittent stress drops, reaching a maximum steadystate stress of $\sim 800 \mathrm{MPa}$.

Geometrically, the pure Mg pillar under c-axis compression undergoes massive basal sliding, resulting in a strong misalignment between the specimen and loading axes. Few instances of traces 


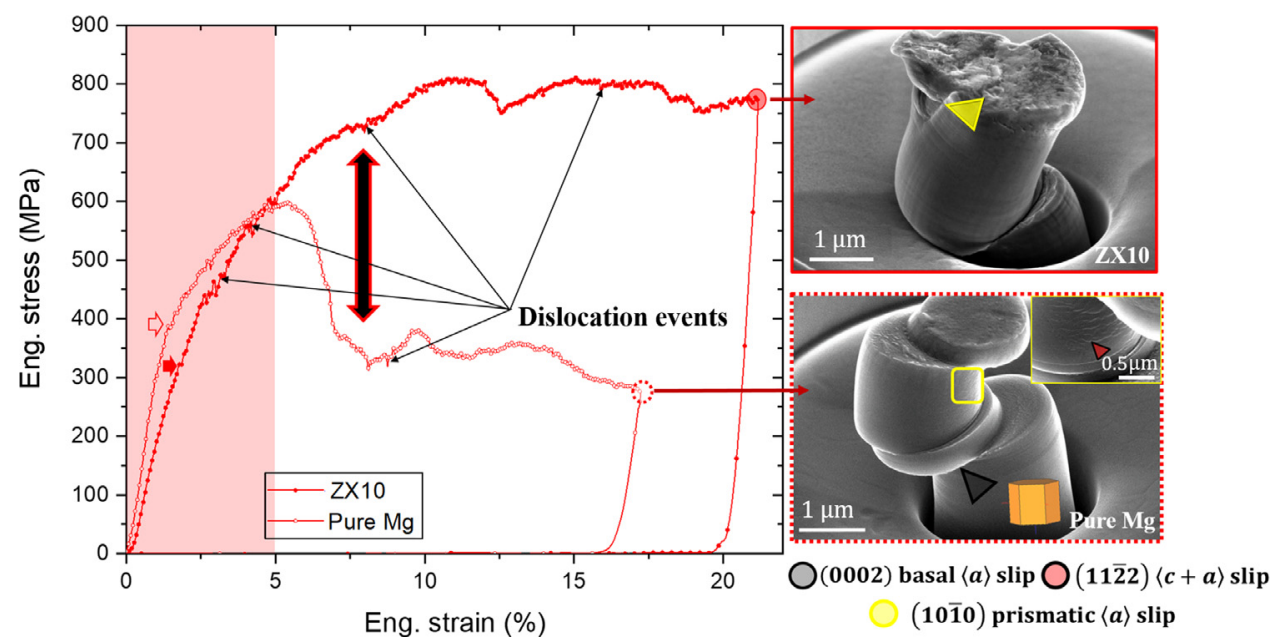

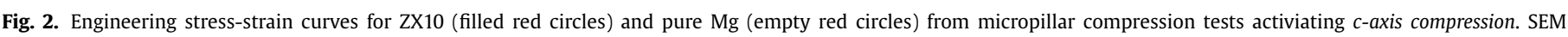

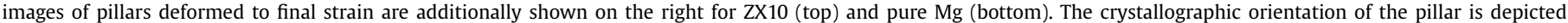

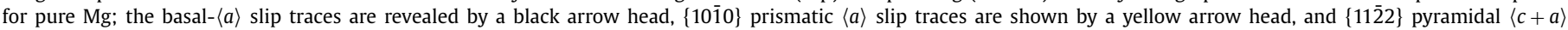
slip traces are shown by a red one (see inset).

along the non-basal planes were also observed. An SEM micrograph of the as-deformed ZX10 micropillar illustrates significantly restrained lateral sliding, corroborating the greater work hardenability in the alloyed specimen compared to pure $\mathrm{Mg}$ (see movie shown in SupplementaryFig. 1). Conversely, the observed pillar distortion in ZX10 most likely arises from uniform basal and nonbasal slip operation. In addition, micro-cracks originating at the top surface and propagating into the pillar (details can be seen in Supplementary Fig. 1) are observed along the prismatic planes (highlighted by the yellow arrow head in Fig. 2).

Fig. 3 shows snapshots taken in situ in the SEM at different strains for pure Mg and ZX10 under the two different loading conditions, to illustrate the evolution of the micropillar geometry as a function of deformation strain. For a detailed view on the deformation progression, the reader is referred to the movie shown in Supplementary Fig. 1. Under c-axis compression, the onset of basalsliding-induced plastic instability occurs in the pure $\mathrm{Mg}$ micropillar when the deformation strain increases from $5 \%$ to $10 \%$. This event clearly correlates with the large stress drop in the stressstrain data shown in Fig. 2, which occurs for strains larger than 5\%. In comparison, the ZX10 micropillar does not reveal massive distortion in the pillar geometry until a deformation strain of $10 \%$, corroborating the significantly higher work-hardening response seen in the stress-strain curve.

In the case of c-axis extension, both the Mg and ZX10 pillar do not reveal any slip traces up to a deformation strain of $5 \%$, which is attributed to the initial twinning-mediated plasticity response for both cases. At larger strains, massive basal shear steps are observed in pure $\mathrm{Mg}$ (already visible at a strain level of $\varepsilon \approx 10 \%$ ), whereas the appearance of discrete basal slip steps is delayed to $15 \%$ strain in the case of the ZX10 alloy. Comparing this with the stress-strain curves under $c$-axis extension, the pillar-shape evolution agrees well with the transition from twinning-mediated to slip-mediated plasticity for strains greater than $7 \%$.

\subsection{Strain-based microstructural evolution of ZX10 under c-axis extension}

In order to assess the local plasticity mechanisms in the ZX10 alloy as a function of imposed plastic strain, microstructural investigations using correlative transmission Kikuchi diffraction and electron microscopy were performed along the longitudinal crosssection of the pillars. The microstructural characterization was fo- cused on the alloyed specimen, because of its superior mechanical performance compared to pure Mg.

Fig. 4 illustrates the strain-based deformation sub-structure evolution under c-axis extension. At a strain of 3\%, the SEM image indicates that the mid-section of the pillar has undergone deformation twinning (see Fig. 4a). The corresponding orientation map of the cross-section (highlighted by the dotted rectangle in Fig. $4 a$ ) reveals the twin to be a $\{10 \overline{1} 2\}$ tension twin, which reorients the $c$-axis of the parent grain nearly parallel to the loading axis. Fig. 4b shows the twinned grain in red in an inverse pole figure orientation map and single-orientation scatter pole figure. The nucleated twin is a well-known high Schmid-factor twin variant, which mostly dominates twinning in pure Mg. Fig. $4 \mathrm{~b}$ also presents the kernel average misorientation (KAM) map for the region in the vicinity of the twin-parent interface. The KAM values are calculated for the second-nearest neighbour assuming an angular threshold of $2^{\circ}$ [41]. The appearance of greenish-yellow colors in the vicinity of the twin-parent interface indicates strain accumulation at the boundaries due to dislocation-twin boundary interactions. In addition, the parent orientation (shown in red in Fig. 4b) undergoes prismatic slip-induced rotation around the $c$ axis and thus deviates from the starting orientation of $\langle 10 \overline{1} 0\rangle \| \mathrm{CD}$ represented in blue (see legend in Fig. 4). An in-grain misorientation axis (IGMA) distribution map shown in Supplementary Fig. 2 shows that the axial spread inside the parent lies in between the $\langle 10 \overline{1} 0\rangle$ and $\langle 11 \overline{2} 0\rangle$ directions, thus indicating that the in-grain rotation occurs about the $c$-axis. Fig. 4c presents dark-field TEM micrographs of the micropillar, revealing the dislocation structures in the twinned and parent grains. The twinned area exhibits a large density of sharp and narrow streaks that look like basal stacking faults arranged in a layered fashion, with many prismatic and pyramidal I dislocation segments enclosed between adjacent faults, which are observed near the twin interface. These observations are further validated by the image quality map (see inset), showing slip traces corresponding to prismatic (shown by a yellow arrow in Fig. 4c) and pyramidal I planes (shown by light blue arrows in Fig. 4c). The twin-parent interface reveals a complex dislocation structure that may relate to the interaction of stacking faults with the twin boundary.

As the deformation strain increases to $6.5 \%$, the twinned crystal expands to occupy the majority of the pillar (see Fig. $4 \mathrm{~d}$ and Fig. 4e). The white dotted frame in Fig. 4d indicates the plane of 


\section{Pure Mg}
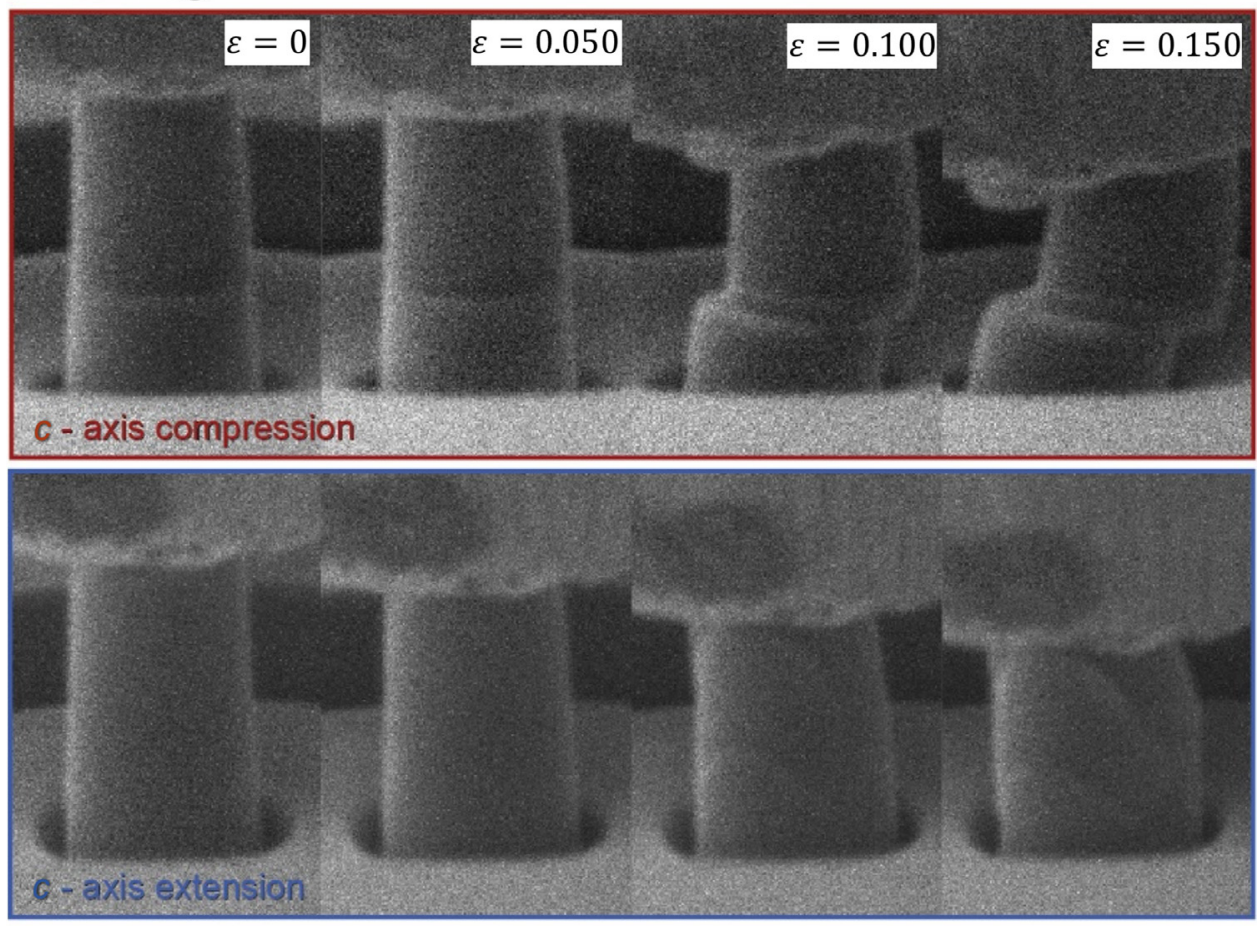

\section{$\mathrm{Mg} Z \mathrm{ZX10}$}
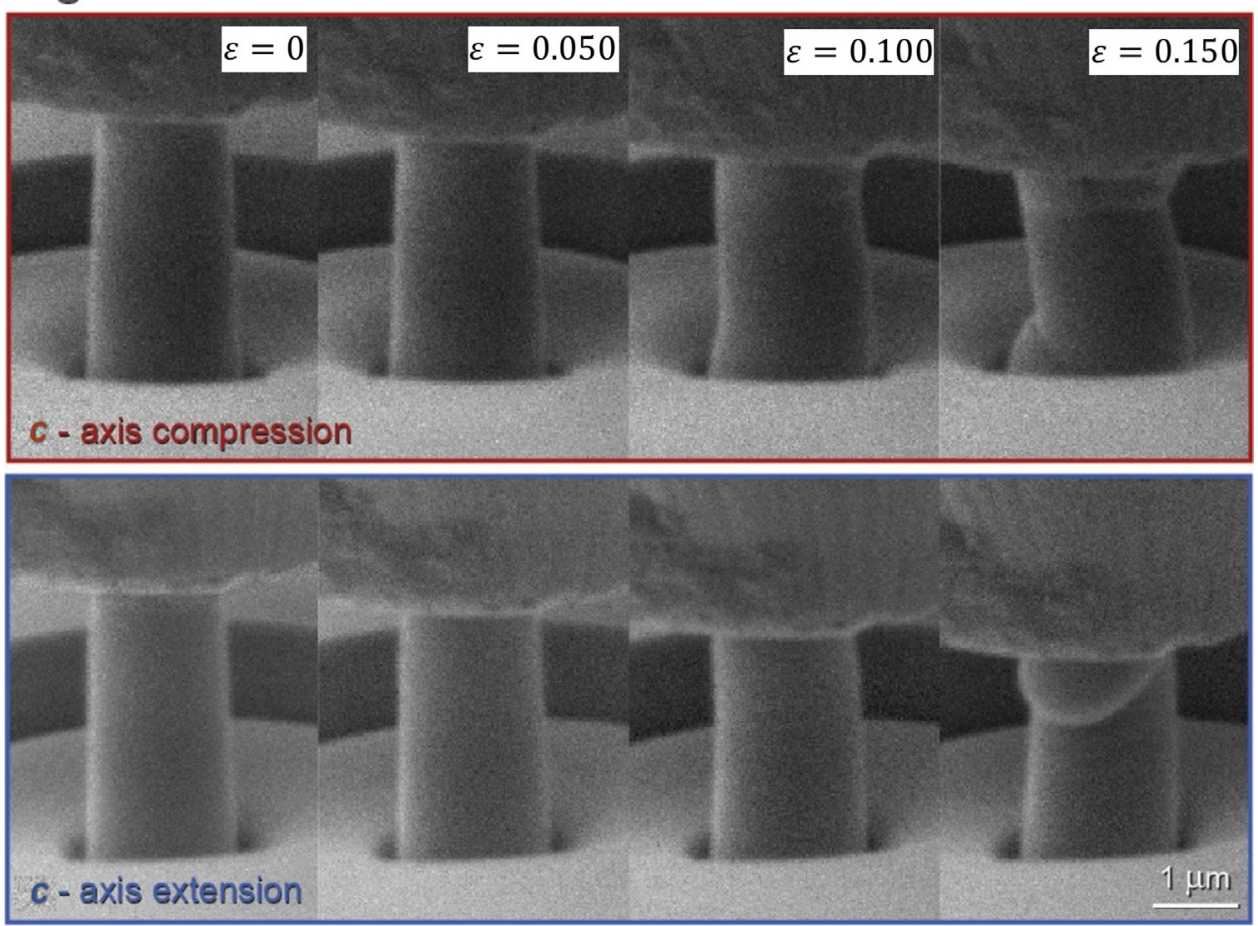

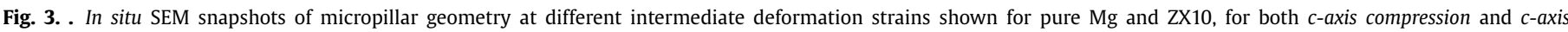
extension.

observation for the t-EBSD and TEM analyses. Simultaneously the twinned grain also exhibits deformation via dislocation slip, where the $c$-axis of the twin starts to rotate away from the compression direction, indicative of non-basal slip activity. Strain accumulation at the twin-parent interface significantly escalates (as shown in Supplementary Fig. 3), which nucleates low Schmid-factor twin variants represented in greenish-yellow color in the inverse pole figure and single-orientation scatter plots of Fig. 4e (marked by white arrow heads). Corresponding TEM micrographs of the twin boundary (see Fig. 4f) indeed reveal the appearance of nano-scale twins (as confirmed by the overlapping diffraction patterns of the parent indicated by a white arrow and the nano-twin shown by a black arrow in Fig. 4f), and thus corroborate such plasticity mechanism to occur in the ZX10 alloy. 


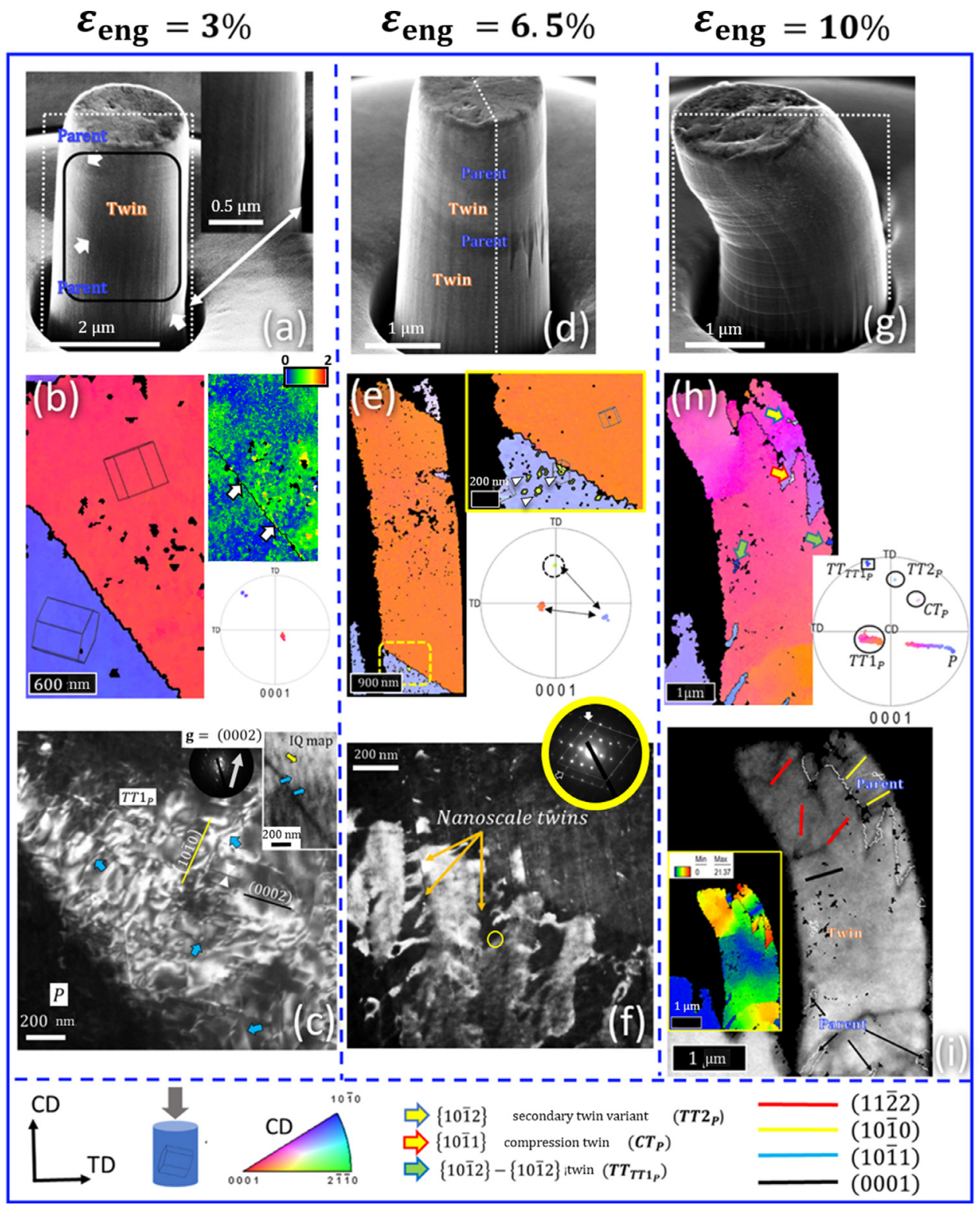

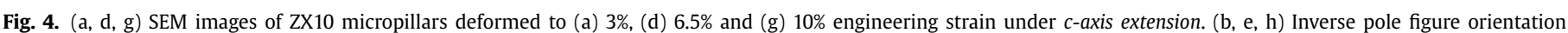

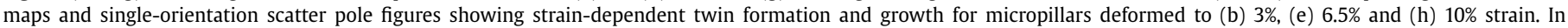

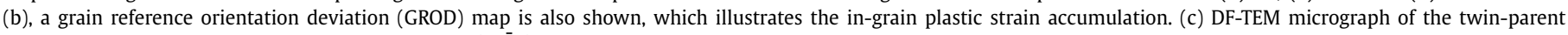

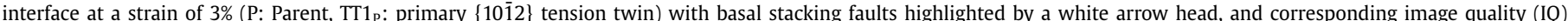

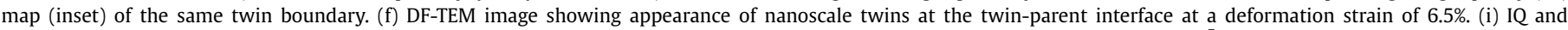

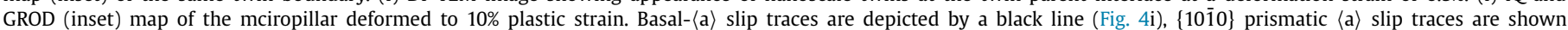

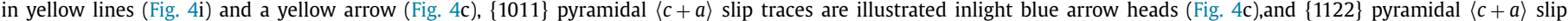
traces are shown in red lines (Fig. 4i). The legend indicates the loading mode with respect to the pillar orientation and starting grain orientation.

On further straining to $10 \%$, the SEM image of the ZX10 micropillar (Fig. 4g) reveals a series of small basal shear steps, in contrast to the massive basal slip seen in pure Mg. The corresponding t-EBSD image (Fig. 4h) of the highlighted cross-section in Fig. 4g indicates that the entire pillar has undergone twinning, extending to even below the base of the pillar, and that significant dislocation plasticity has also occurred. Large orientation gradients inside the twin indicates a strong rotation of the $c$-axis away from the compression direction, typically associated with $\langle c+a\rangle$ slip activiation (see Fig. 4h). On the other hand, the remaining untwinned parent grain in the upper right of the pillar also shows complex dislocation activity as revealed by the large orientation spread seen in the single-orientation scatter pole figure. Slip-trace analysis on the image quality (IQ) dataset of the investigated area indeed reveals acti- vation of pyramidal II $\langle c+a\rangle$ slip inside the twin and prismatic slip inside the parent grain. The grain reference orientation deviation (see GROD map shown as inset) for the micropillar indicates local stress concentration primarily at the top edge of the pillar, bottom right edge near the pillar base, and near the twin-parent interface. Apart from dislocation slip, the twinned grain further undergoes tension twinning, giving rise to $\{10 \overline{1} 2\}-\{10 \overline{1} 2\}$ double tension twins (highlighted by the green filled arrows with blue border in Fig. 4h). On the other hand, low Schmid-factor twin variants are also observed inside the untwinned parent in the top right corner of the micropillar, shown by the yellow filled arrow with blue border (see Fig. 4h). The increasing stress that builds up at the twinparent interface also results in the nucleation of nanoscale com- 


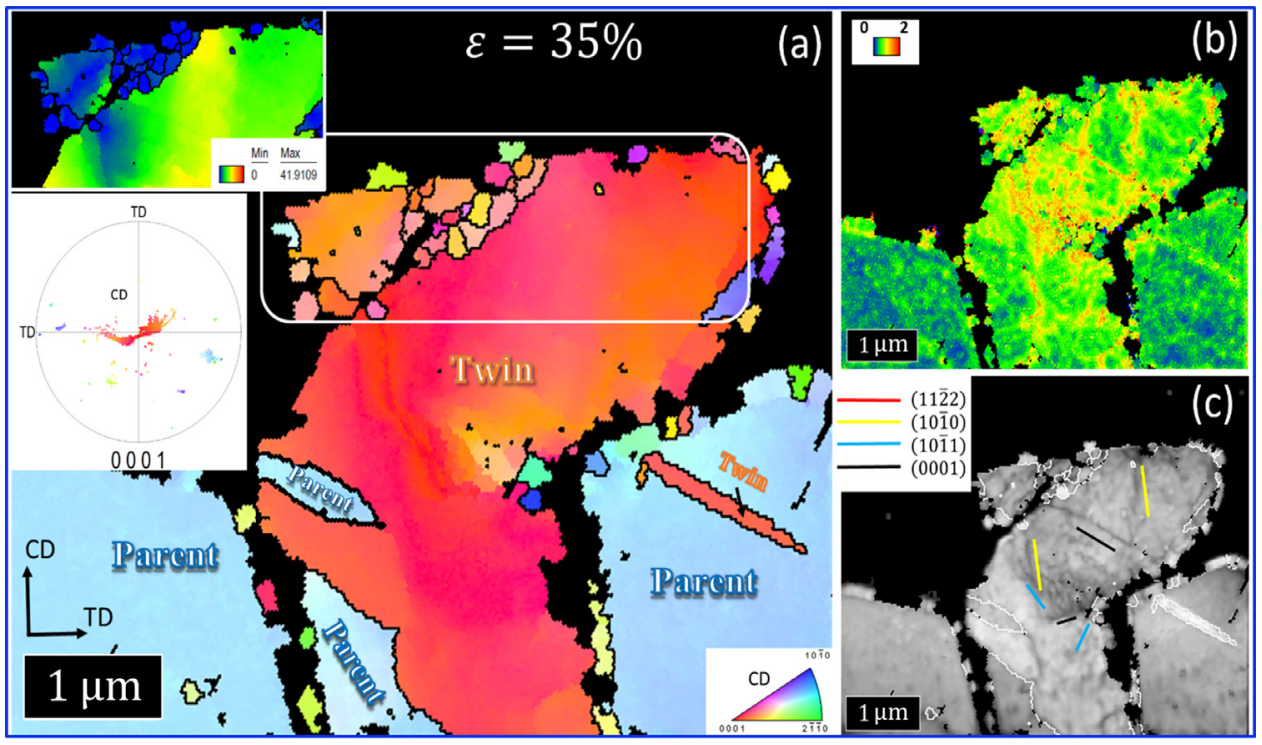

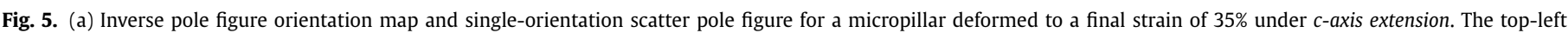

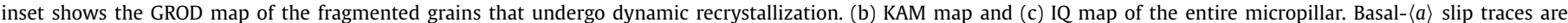
depicted in black, $\{10 \overline{1} 0\}$ prismatic $\langle a\rangle$ slip traces are shown in yellow, $\{10 \overline{1} 1\}$ pyramidal $\langle c+a\rangle$ slip traces are illustrated in light blue.

pression twins, as highlighted in Fig. $4 \mathrm{~h}$ by a yellow filled arrow with red border.

Fig. 5 presents the t-EBSD analysis of the micropillar strained to a final deformation strain of 35\%. Fig. 5a displays the inverse pole figure map of the deformed pillar. The twinned pillar exhibits a large orientation spread and a significant dislocation substructure, whereby the identity of the original twin orientation is completely destroyed, as depicted by the single-orientation scatter plot. The latter indicates tilting of the $c$-axis away from the $C D$ towards the TD. The top section of the pillar illustrates severe grain fragmentation, generating strain-free ultrafine grains. Fig. 5b shows the corresponding KAM map, where the local strain appears to systematically accumulate along the identified slip traces (see IQ map in Fig. 5c), which correspond to basal, prismatic and pyramidal I traces.

\subsection{Strain-based microstructural evolution of ZX10 under c-axis compression}

Fig. 6 gives an overview of the strain-dependent microstructural development under c-axis compression. The top row presents SEM images of micropillars compressed to different strain values of 3\%, $6 \%$ and $8.5 \%$ (see Figs. 6a, 6d and $6 \mathrm{~g}$ ). The images indicate larger slip offsets at the pillar surface to occur with increasing deformation strain, associated with a progressive increase in the net basal shear accumulation. The corresponding t-EBSD maps, displayed in inverse pole-figure color coding in the middle row (see Figs. 6b, 6e and $6 \mathrm{~h}$ ), reveal the absence of twinning in the pillars, suggesting the plasticity to be borne solely by dislocation slip. The comparison of the alignment of the $c$-axis upon increasing the strain from $3 \%$ to $8.5 \%$ reveals a gradual rotation of the basal planes away from the compression plane. The inset images in Fig. $6 \mathrm{~b}$, corresponding to IQ t-EBSD data and a bright-field TEM micrograph, reveal simultaneous basal, prismatic and pyramidal II $\langle c+a\rangle$ slip. At a strain of $8.5 \%$, the IQ map (Fig. $6 \mathrm{~h}$ ) reveals sharp slip bands along the primatic planes, and the corresponding KAM data (Fig. 6h) indicates significant stress build-up along the slip bands. The bottom row presents TEM micrographs of the entire pillar for the selected deformation strains. At 3\% strain (Fig. 6c) the dislocation activity appears to be primarily confined to the lower half of the pillar and to the top corners. As the strain increases to 6\% (Fig. 6f), the dislocation activity extends to the top half of the pillar, described by dislocation traces along basal and non-basal planes. A further strain increment gives rise to a more complex dislocation structure (Fig. 6i) across the entire pillar with the activation of multiple slip modes that may mutually interact.

\subsection{Defect analysis using transmission electron microscopy}

An in-depth local-scale analysis of the dislocations and defect structures is realized by TEM. Figs. $7 \mathrm{a}$ and $7 \mathrm{~b}$ show TEM micrographs of the micropillar deformed under c-axis extension up to a strain of $6.5 \%$, revealing the structure of the twinned grain and the remaining parent. The beam direction for the twinned area was parallel to $\langle 10 \overline{1} 0\rangle$. A two-beam condition was established for the twinned grain with diffraction vectorsg $g_{1}=(0002)$ and $\mathbf{g}_{2}=$ $(11 \overline{2} 0)$ (see Fig. 7a). The light grey arrows indicate the directions of the operating diffraction vector. For each diffraction vector, the images were acquired under weak-beam dark-field (WBDF) conditions, complying to a $\mathbf{g}$. (3g)diffraction. Fig. 7a reveals a large density of (0002) defects traversing across the pillar width (indicated by white arrow heads) and appear as straight, narrow streaks. The speckled diffraction contrast along these sharp planar streaks in Fig. 7a most likely indicates that these are $I_{2}$-type 'deformation' faults that are aligned in an edge-on view, presumably created by the glide of Shockley-type partials, i.e. $\left(\frac{1}{3}\langle 10 \overline{1} 0\rangle+\frac{1}{3}\langle 01 \overline{1} 0\rangle\right)$ during plastic deformation. In between the stacking-fault like defects, there exists a large density of $\langle c\rangle$ dislocation segments lying on the non-basal pyramidal II planes (shown by red arrows in Fig. 7a). Imaging of the same region under $\mathbf{g}_{2}=(11 \overline{2} 0)$ reveals only $\langle a\rangle$ dislocation segments based upon the $\mathbf{g} \cdot \mathbf{b}$ invisibility criterion, thereby confirming that the dislocations on the pyramidal planes possess a $\langle c+a\rangle$ Burgers vector.

Fig. $7 c$ presents the initial parent grain with an operating diffraction vectorg $=(0002)$. The image once again reveals dislocation segments that have a $\langle c+a\rangle$-type Burgers vector on pyramidal II glide planes in the vicinity of the twin-parent interface. The inset image (Fig. 7d) presents a zoomed-in view of the twin boundary, revealing half-dislocation loops pinned to the twin boundary, with the dislocation line bowing out into the parent grain, which is a typical feature of a Frank-Read source. Such sources can potentially give rise to dynamic dislocation nucleation during deformation. 


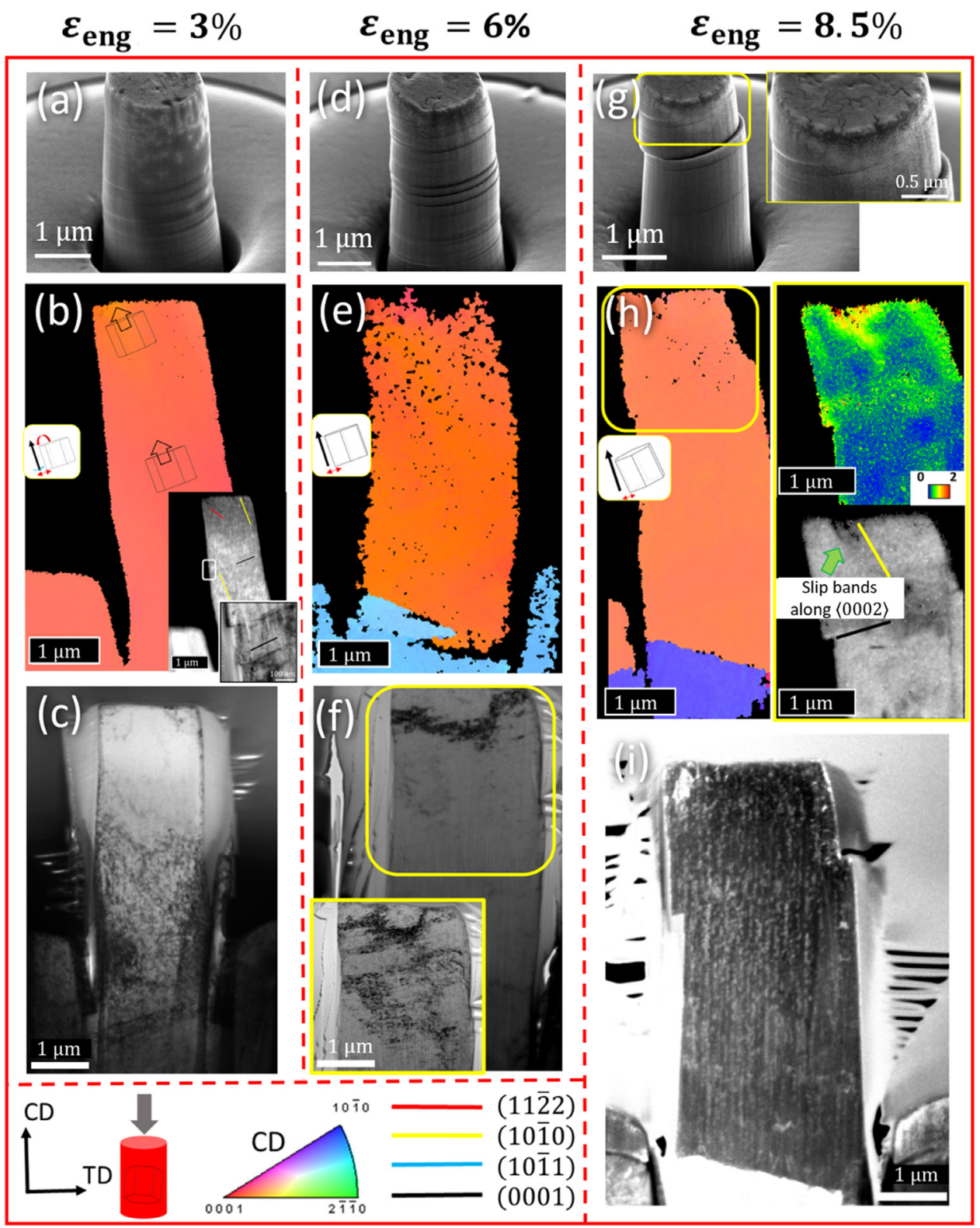

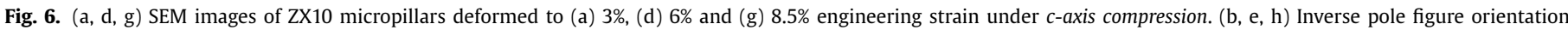

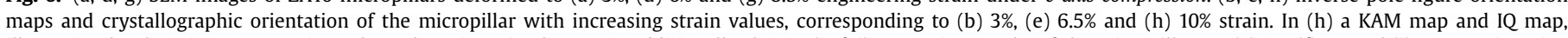

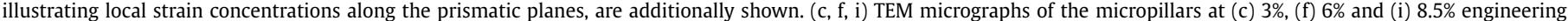

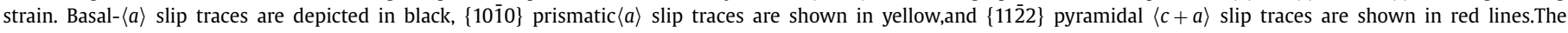
legend indicates the loading mode with respect to the pillar orientation and starting grain orientation.

In order to confirm the presence of stacking faults, the lamella was tilted further until the edge-on faults appeared as bright and dark fringes as shown by the white arrow heads in the BF-TEM image in Fig. 8a. The operating diffraction vector corresponds to $\mathbf{g}=(10 \overline{1} 1)$. The faint contrast of the Shockley partial dislocations is denoted by the thick black arrows. A large number of curved $\langle c+a\rangle$ dislocations are additionally observed to interact with the stacking faults (denoted by red arrow heads in Fig. 8a). Fig. 8b shows a schematic illustration of the TEM image in Fig. 8a, with the thick black arrows showing the bounding partial dislocations. The red curved lines in Fig. 8b correspond to pyramidal dislocations. Fig. 8c shows a TEM image simulation performed for a basal stacking fault observed under similar imaging conditions as those in Fig. 8a. A foil normal corresponding to $\langle 10 \overline{1} 0\rangle$ was chosen, with the beam direction close to $\langle\overline{7} \overline{2} \overline{5} 3\rangle$. The operating diffraction vector was chosen as (10ī1). The leading and trailing Shockley par- tials were defined by the Burgers vector $\frac{1}{3}\langle 10 \overline{1} 0\rangle$ and $\frac{1}{3}\langle 01 \overline{1} 0\rangle$, respectively. The fault-plane normal corresponded to $\langle 0002\rangle$, and a lamella thickness of $250 \mathrm{~nm}$ was chosen for the simulation. The good agreement between the experimental and simulated images suggests that the observed planar defects are indeed $I_{2}$-type 'deformation' faults.

Fig. 9 presents TEM micrographs of a micropillar deformed under c-axis compression to a strain of 6\%. Figs. 9a-c are imaged with an operating diffraction vector $\mathbf{g}_{1}=(0002)$, where Fig. 9c is acquired under WBDF diffraction conditions $(\mathbf{g} \cdot(3 \mathbf{g}))$. Similar to the twinned grain in Fig. 7a, the pillar comprises a large density of (0002) stacking faults with pyramidal II $\langle c+a\rangle$ dislocation segments. Fig. 9d shows a bright-field (BF) image of the same area as in Fig. 9c. The diffraction contrast reveals small prismatic dislocation loops visible at the intersection zones of the non-basal dislocation segments and $I_{2}$-type basal stacking faults. 


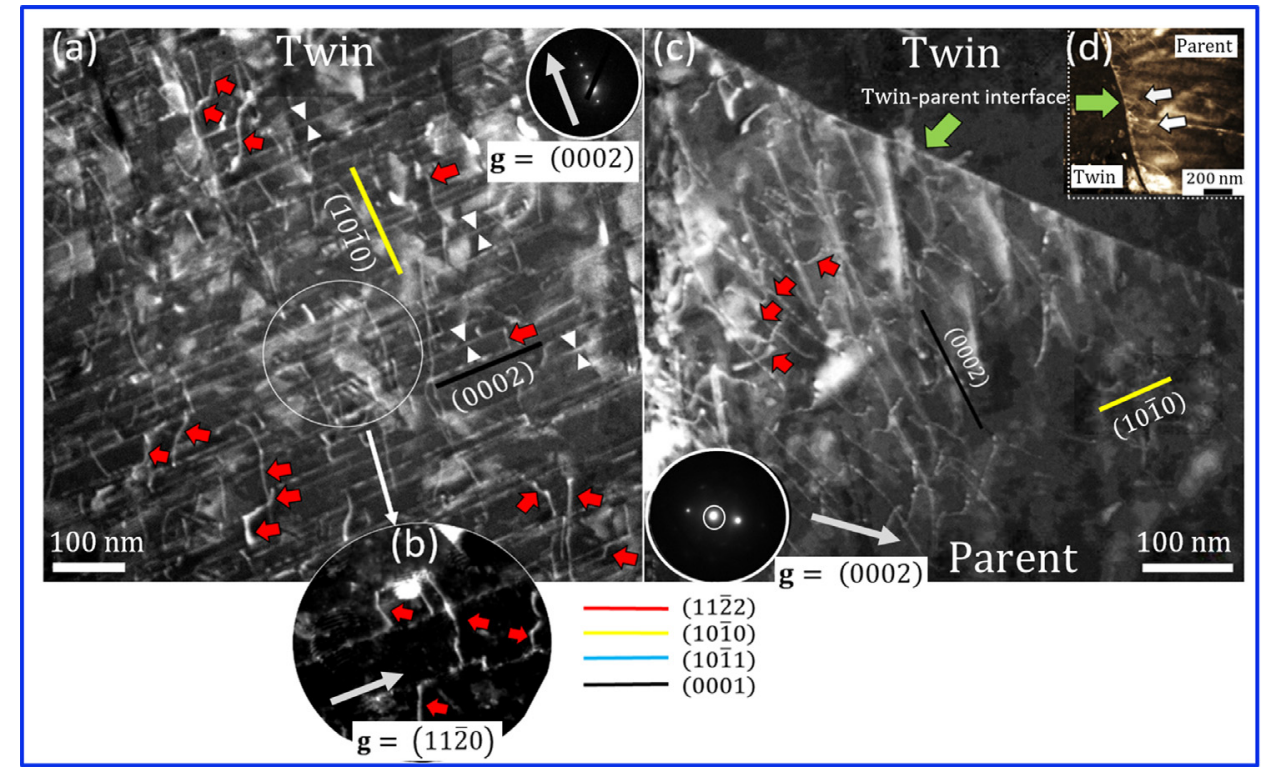

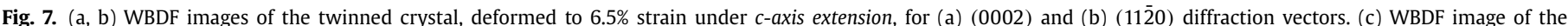

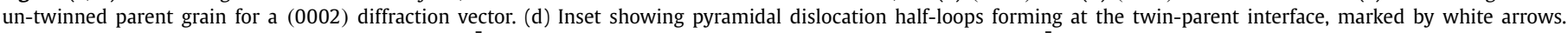

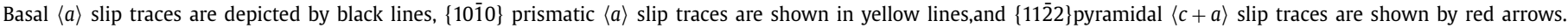
The white arrow heads highlight basal stacking faults.

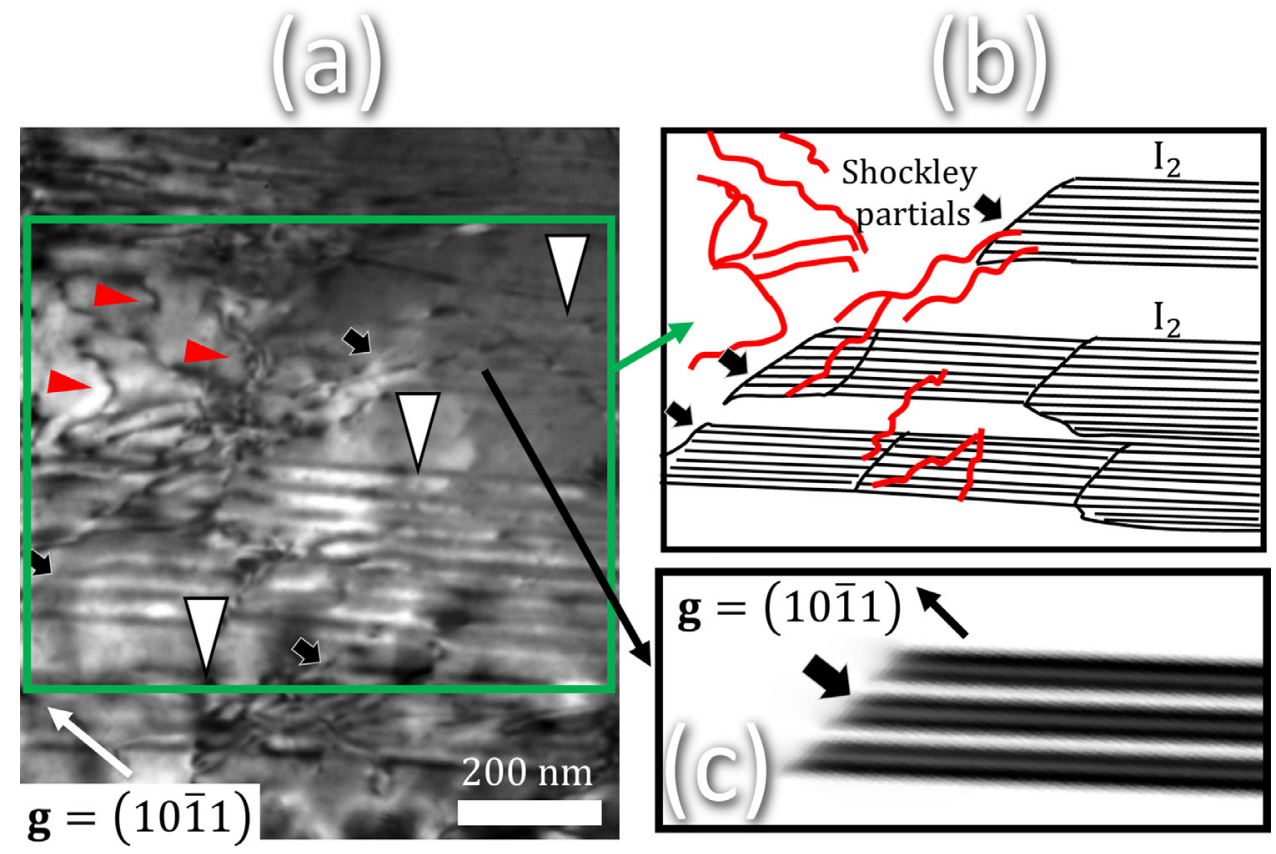

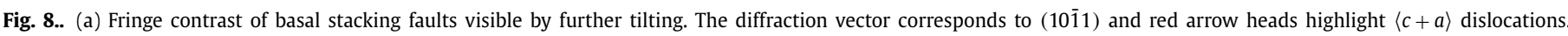

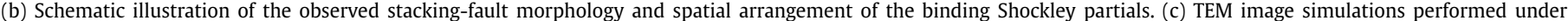
the same imaging conditions, illustrating the presence of $I_{2}$-type 'deformation' faults.

\section{Discussion}

4.1. Twinning characteristics during c-axis extension: ZX10 versus pure $\mathrm{Mg}$

The loading of the ZX10 micropillars normal to their c-axis resulted in a combination of twinning and dislocation slip-induced plasticity response. Pillar deformation that proceeded by means of $\{10 \overline{1} 2\}$ tension twinning resulted in a characteristic $86^{\circ}$ rotation of the $c$-axis and aligned it nearly parallel to the compression axis. Theoretically, a given orientation when compressed perpendicu- lar to its $c$-axis can potentially twin along six crystallographically equivalent $\{10 \overline{1} 2\}$ planes [42]. However, in practical cases only those twin orientations whose resolved shear stress on the habit plane in the twinning shear direction exceeds a critical value will nucleate, i.e. those possesing the highest Schmid factor $\left(m_{\text {global }}\right)$, as illustrated in Figs. 10a and 10b. In the present case, the twin orientation nucleating at 3\% deformation strain corresponds to the highest Schmid-factor variant, as indicated in Fig. 10a.

Subsequent straining to $6.5 \%$, however, promoted additional twinning events in the form of nano-scale twins appearing at the twin-parent interface (Figs. 4e and 4f), complying with the low 


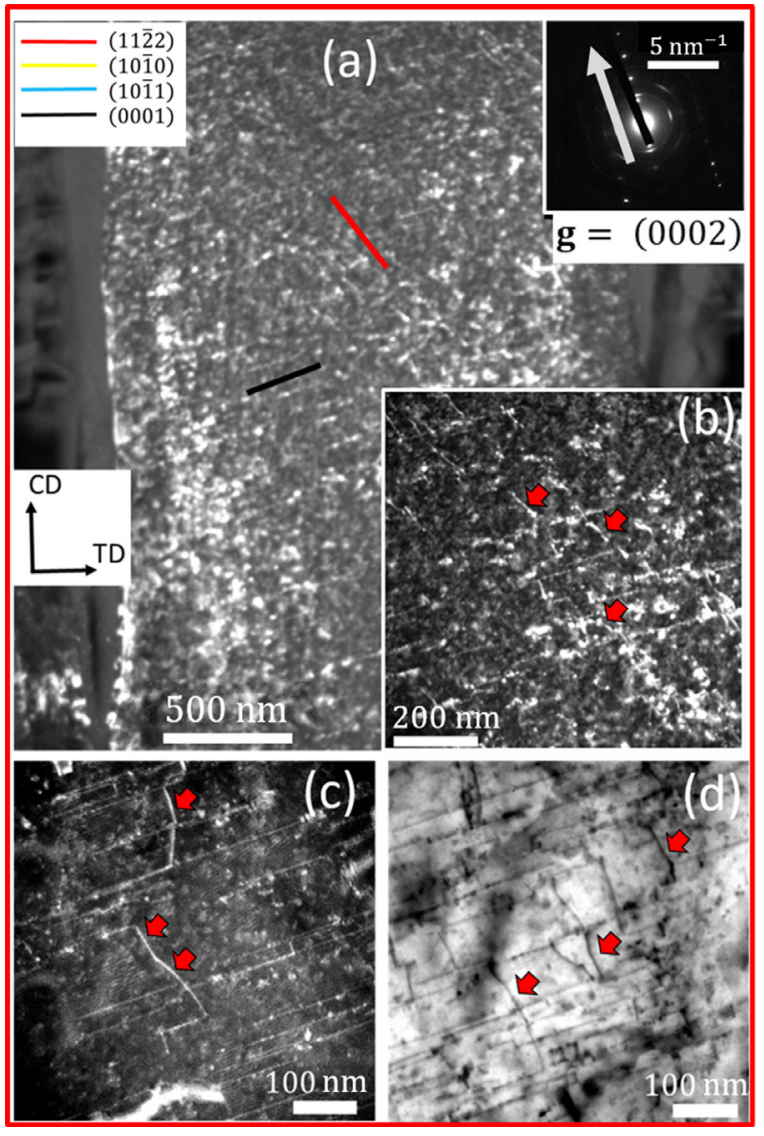

Fig. 9. (a, b) DF TEM images of the micropillar at different magnifications for (0002) diffraction. Correlative (c) WBDF and (d) BF images of a representative region in the micropillar deformed to $6 \%$ engineering strain under c-axis compression. Basal $\langle a\rangle$ slip traces are depicted by black lineand $\{11 \overline{2} 2\}$ pyramidal $\langle c+a\rangle$ slip traces are shown by red arrows (9b, 9c and 9d) and a red line (9a).

Schmid-factor twin variants. These twin variants theoretically correspond to a Schmid factor of 0.12 as opposed to the red-colored primary twin variant that has a Schmid factor of 0.499. Such 'nonSchmid' twinning response stems from the dominant role of local stress heterogeneities or strain-accommodation requirements near pre-existing defect structures, such as grain boundaries. In this regard, a critical factor that influences the aforementioned Schmid rule for twin-variant selection in hexagonal materials is the ease of accommodation of associated twinning shear via slip or twinning in the neighboring grain. In order to assess such local strain-accommodation effects on twin nucleation, the role of grainboundary crystallography in terms of strain transmission needs to be evaluated. In the case of metallic materials the feasibility of strain transmission across grain boundaries is determined by the geometric alignment of the active slip/twinning systems across the interface and the minimization of the dislocation energy at the boundary [41,43-45]. Mathematically, it can be described by a local Schmid-factor parameter, $m_{\text {local }}$, given as

$m_{\text {local }}=\left(\mathbf{n}_{1} \cdot \mathbf{n}_{2}\right)\left(\mathbf{b}_{1} \cdot \mathbf{b}_{2}\right)$,

where $\mathbf{n}_{1}$ and $\mathbf{n}_{2}$ are the normalized intersection lines common to the slip/twin planes and boundary plane, and $\mathbf{b}_{1}$ and $\mathbf{b}_{2}$ are the normalized twinning shear/slip directions in the pile-up and emission grains. Larger values of $m_{\text {local }}$ indicate well aligned incoming and outgoing deformation modes across the grain boundary, and therefore easier strain transmission. Potential twin variants, for which the active slip systems in the adjacent grain are able to eas- ily accommodate resultant shear because of their higher $m_{\text {local }}$, can thus be favored despite having lower $m_{\text {global }}$ values.

Investigations using t-EBSD of the twinned regions in Fig. 4 indicate the activation of both basal slip and non-basal dislocations on pyramidal planes. Fig. 10c presents the quantification of the local Schmid factor $\left(m_{\text {local }}\right)$ across the twin-parent interface for different combinations of incoming and outgoing slip/twinning modes, which were experimentally identified in the deformed ZX10 micropillars. Three alignment scenarios across the twinparent interface were evaluated, i.e.basal $\langle a\rangle$ slip $-\{1012\}$ tension twinning, basal $\langle a\rangle$ - basal $\langle a\rangle$ slip, and pyramidal II $\langle c+a\rangle$ slip $\{10 \overline{1} 2\}$ tension twinning. For the transition of pyramidal II $\langle c+a\rangle$ slip - $\{10 \overline{1} 2\}$ tension twinning across the twin boundary, high $m_{\text {local }}$ values indicate that the activation of pyramidal II $\langle c+a\rangle$ slip in the twinned grain can generate low Schmid-factor twin variants, as shown in greenish-yellow for the parent in Fig. 4e. Conversely, when considering only basal-slip activation, such a nonSchmid twinning event is unlikely, as revealed by the low $m_{\text {local }}$ values for such a case. Moreover, low $m_{\text {local }}$ values in the case of basal slip as the sole deformation mode in both the twin and parent grains emphasize upon the role of twin boundaries in acting as strong barriers against the motion of basal dislocations and thus give rise to stress pile-up at the interface. This aspect with respect to slip dynamics is discussed in greater detail in Section 4.3.

At even higher strains $(\geq 10 \%)$, twinning extends across the whole pillar, giving rise to a transition into dislocation-slip mediated plasticity. The simultaneous activity of basal and non-basal slip gives rise to complex dislocation-twin boundary interactions, where the local stress fields facilitate nucleation of $\{10 \overline{1} 1\}$ compression twins at the twin-parent interface. Additionally, the appearance of $\{10 \overline{1} 2\}$ nano-twins inside the already twinned grains are also observed. Nucleation of such second-generation twins is possible due to sufficient non-basal slip activity inside the twinned grains, which realign the $c$-axis away from the $\mathrm{CD}$ and enable such secondary twinning events.

In comparison, the twinning characteristics in pure $\mathrm{Mg}$ indicated strong variant selection resulting in the appearance of two twin variants in the deformed $\mathrm{Mg}$ micropillar. On comparison with the possible twin orientations (shown in Fig. 10), the two twin variants correspond to orientations close to the orange-red, high Schmid-factor variants that typically originate from the green $\langle 11 \overline{2} 0\rangle \| \mathrm{CD}$ parent (see Fig. 10b). EBSD analysis on the original orientation of the pure $\mathrm{Mg}$ pillar indicates that the parent grain deviated by $\sim 10-15^{\circ}$ from the ideal $\langle 10 \overline{1} 0\rangle|| C D$ orientation (see Supplementary Fig. 4). SEM investigations on the parent grain also indicate traces of prismatic $\langle a\rangle$ slip (see Fig. 1). It is thus proposed that the simultaneous activity of prismatic slip along with primary tension twinning in the parent grain promotes rotation towards the more stable $\langle 11 \overline{2} 0\rangle \| \mathrm{CD}$ orientation, thereby explaining the presence of two high Schmid-factor twin variants. Subsequent deformation inside the twins proceeds via profuse basal slip.

\subsection{Effect of $\mathrm{Zn}$ and $\mathrm{Ca}$ solutes on twinning stresses: Nucleation and growth}

The stress values at which the twins nucleate and grow during the onset of $\{10 \overline{1} 2\}$ twinning deformation are estimated from the loading curves shown in Fig. 1, which highlight the twin nucleation and growth events by green and blue arrows, respectively. Fig. 11a compares the stress-strain behavior corresponding to the twinning onset in ZX10 with that of pure Mg. The observed stress peak prior to the sharp stress drop at around 1.9\% strain is attributed to the stress required to generate a stable twin nucleus bound by $\{10 \overline{1} 2\}\langle 10 \overline{1} 1\rangle$ twinning partials, and the subsequent short stress plateau corresponds to the twin-growth regime. The appearance of the stress plateau relates to an atomic-shuffling dominated lateral 


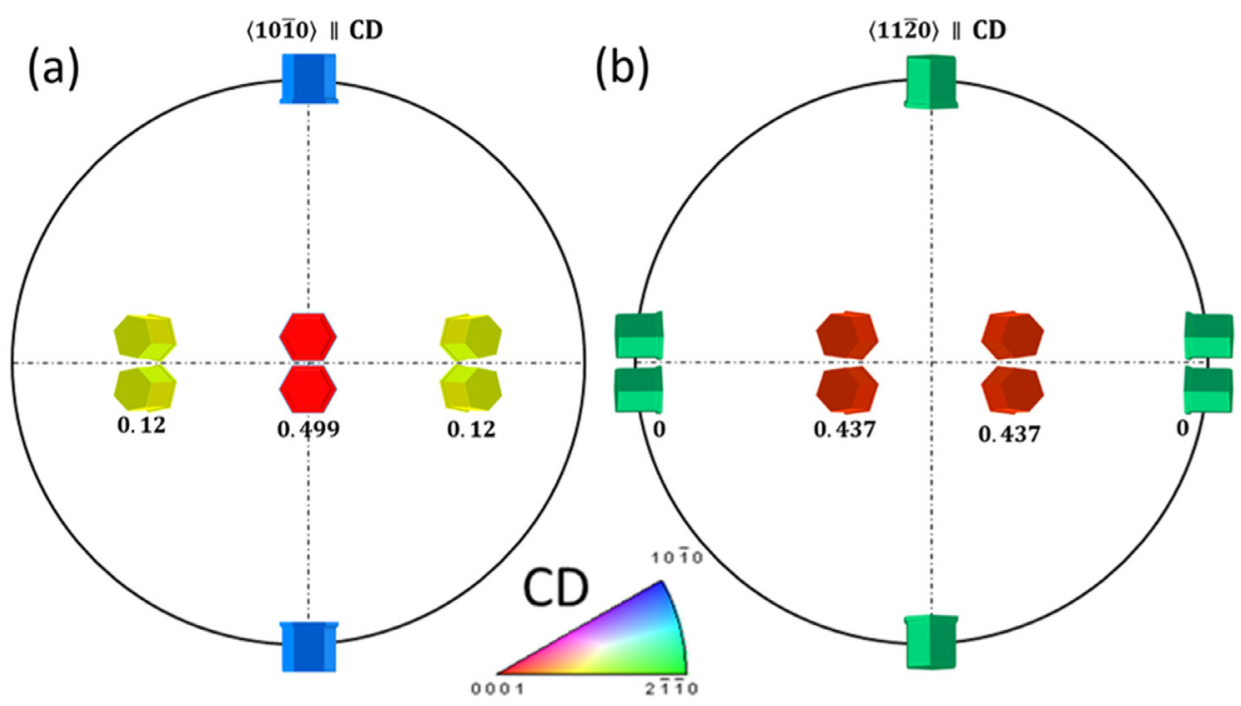

(c)
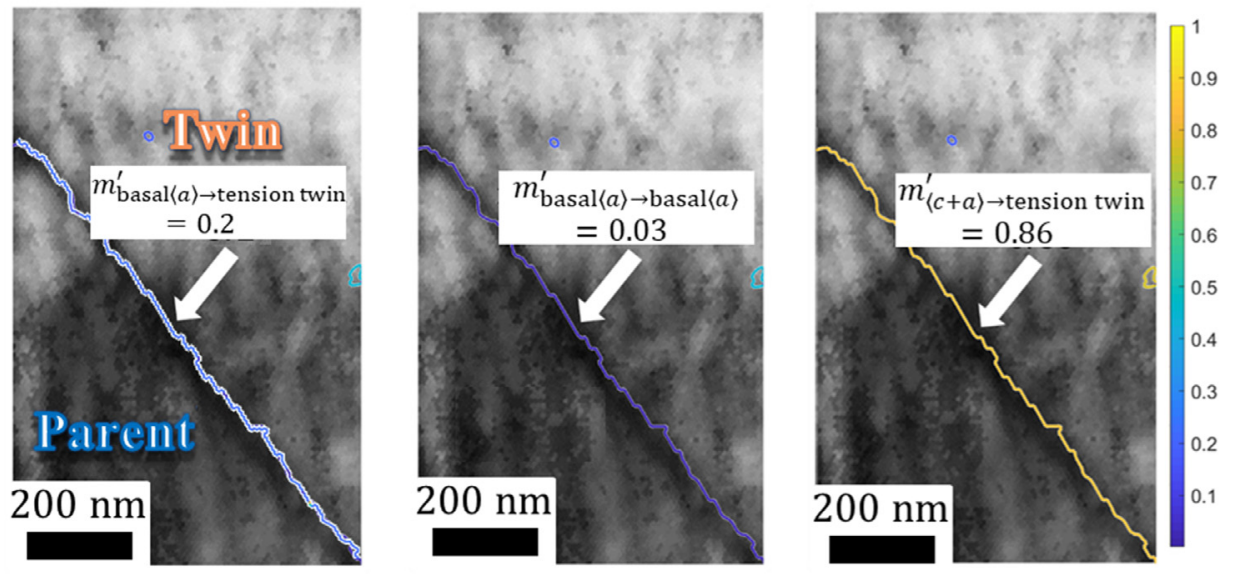

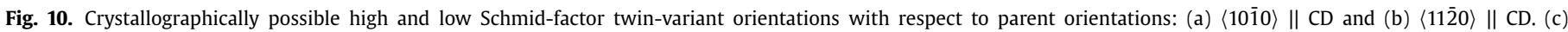

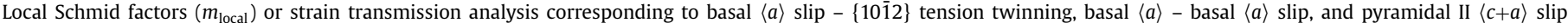
$-\{10 \overline{1} 2\}$ tension twinning.

twin-growth mechanism, which is known to proceed with negligible shear [46]. Fig. $11 \mathrm{~b}$ shows the $\{10 \overline{1} 2\}$ tension-twin nucleation and growth stresses, which correspond to the peak stress and the plateau stress values averaged over 3 independent flow curves, respectively. While the twin-growth stresses are similar for both materials ( $176--178 \mathrm{MPa}$ ), the twin-nucleation stress shows a clear increment in case of ZX10 $\left(\Delta \tau_{\text {rel }}\right.$ twin $\left.\sim 29 \mathrm{MPa}\right)$ as compared to pure $\mathrm{Mg}$ (at $\sim 198 \mathrm{MPa}$ ). This clearly illustrates the benefit of $\mathrm{Zn}$ and $\mathrm{Ca}$ solutes in strengthening the twinning response of $\mathrm{Mg}$.

In order to clearly correlate the influence of solutes on twinning, it is important to investigate the associated atomistic processes that are at play during twin nucleation in hexagonal metals and alloys. Despite the fact that the exact mechanistics of twin nucleation are a topic of debate, there have been several theoretical and modelling efforts to predict the possible pathways of twin formation $[8,9,47,48]$. Based upon energetic considerations and comparisons with experimentally measured twin-boundary energies, the generally agreed mechanism of twin formation is the one involving zonal dislocations, also referred to as zonal-twinning mechanism $[47,49,50]$.
Mathematically [47], the formation of a twinning partial, described by its Burgers vector ' $\mathbf{b}_{\mathbf{t w}} \rightarrow$ ', on the $\{10 \overline{1} 2\}$ twinning plane along the $\langle 10 \overline{1} 1\rangle$ shear direction is given by

$\mathbf{b}_{\mathbf{n} \leftarrow}+\mathbf{b}_{\mathbf{p} \rightarrow}=\mathbf{b}_{\mathrm{tw} \rightarrow}$

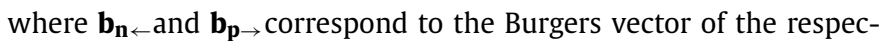
tive partial dislocations with opposite signs that account for the displacements needed for $\{10 \overline{1} 2\}$ planes to change their stacking type, with their magnitudes given as $\left|\mathbf{b}_{\mathbf{n} \leftarrow \mid}\right|=\frac{\gamma^{2}}{\sqrt{3+\gamma^{2}}}$ and $\left|\mathbf{b}_{\mathbf{p} \rightarrow \mid}\right|=$ $\frac{3}{\sqrt{3+\gamma^{2}}}$, and $\gamma=|c / a|$. The magnitude of $\mathbf{b}_{\mathbf{t w} \rightarrow}$ can be expressed as $\left|\mathbf{b}_{\mathbf{t w} \rightarrow}\right|=\left|\mathbf{b}_{\mathbf{p} \rightarrow}\right|-\left|\mathbf{b}_{\mathbf{n} \leftarrow}\right|=\frac{3-\gamma^{2}}{\sqrt{3+\gamma^{2}}}$ and possesses an opposite sign

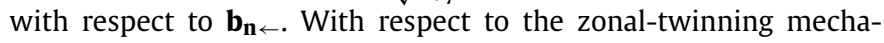
nism, twin nucleation involves the formation of zonal dislocations, which simultaneously comprise the glide of $\mathbf{b}_{\mathbf{n}} \leftarrow$ along the twinparent interface and the atomic shuffling associated with shear, which is induced by multiple twinning partials $\mathbf{b}_{\mathbf{t w} \rightarrow}$ that glide on adjacent $\{10 \overline{1} 2\}$ planes inside the twinned nucleus. The net Burgers vector of a zonal dislocation is then defined as $\mathbf{b}_{\text {zonal }}=$

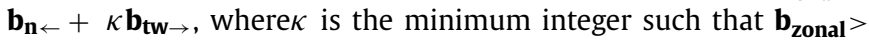




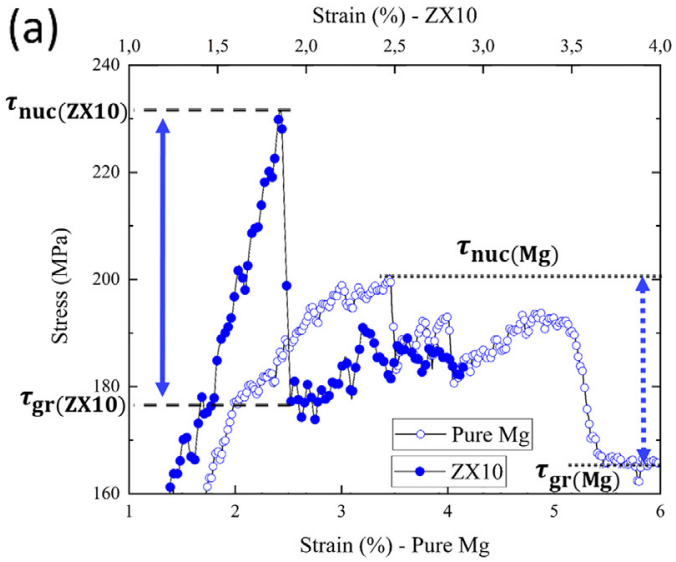

(b)

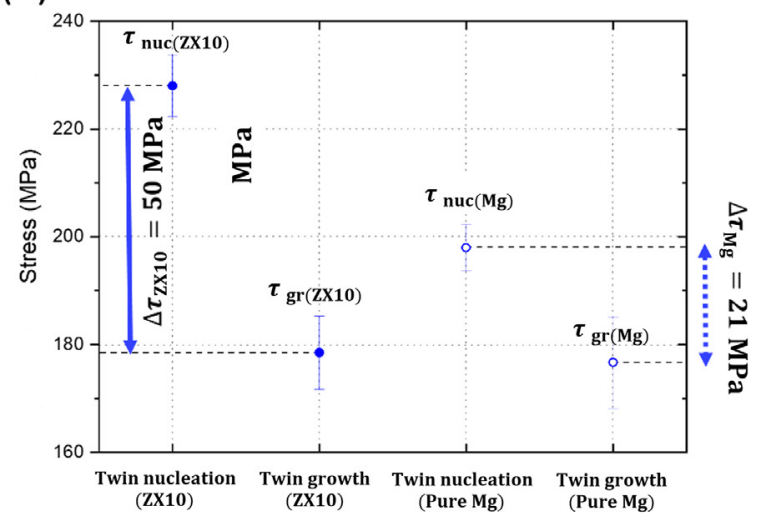

Fig. 11. (a) Characteristic stress-strain response associated with twin nucleation and twin growth in pure Mg (empty blue cricles) and ZX10 (filled blue circles). (b) Experimentally measured averaged twin-nucleation and twin-growth stresses for both materials.

0 for $\gamma<\sqrt{3}$. The corresponding minimum thickness of a twin nucleus stable enough to transition into growth is given by $(2 \kappa+1)$ $\{10 \overline{1} 2\}$ planes.

In the presence of a solute environment, dislocation glide will experience resistance resulting from its interaction with the solute atoms. The nature of this interaction is a combination of elastic effects i.e. the solute and dislocation stress fields mutually interact, and a chemical contribution or Suzuki effect that results from changing the interatomic bonding environment due to segregation of solutes inside dislocation cores and stacking faults [51,52]. Mechanistically, the dislocation line traversing in a field of solutes will adopt a wavy character such that it statistically finds favorable regions of solute fluctuations, thereby lowering the potential energy. However, the bowing of the dislocation line occurs at the cost of additional elastic line energy compared to an otherwise straight dislocation segment. This additional stress component accounts for dislocation strengthening [53].

Considering the sizable strength increment for twinning in ZX10 despite the leanness of its alloying content, conventional solid-solution strengthening cannot be seen as the sole responsible strengthening mechanism, as is the case for a random solid solution. Comparing the atomic radii of $\mathrm{Zn}(134 \mathrm{pm})$ and $\mathrm{Ca}(197 \mathrm{pm})$ with that of $\mathrm{Mg}$ (160 pm), it can be concluded that the atomic size misfits generated by the solutes will give rise to tensile and compressive stress fields, respectively. From the perspective of chemical affinities, the binding energies $(\Delta \mathrm{E})$ for $\mathrm{Mg}-\mathrm{Ca}, \mathrm{Mg}-\mathrm{Zn}$ and $\mathrm{Zn}-$ Ca pairs are $-6 \mathrm{~kJ} \mathrm{~mol}^{-1},-4 \mathrm{~kJ} \mathrm{~mol}^{-1}$ and $-22 \mathrm{~kJ} \mathrm{~mol}^{-1}$, respectively [54,55]. The highly negative values indicate a strong propensity of heterogeneous $\mathrm{Zn}-\mathrm{Ca}$ bond-pair formation in the ZX10 alloy via 'uphill' diffusion. Both their chemical affinity and the reduction in elastic strain-energy contribution due to their positive and negative atomic-size misfits with respect to $\mathrm{Mg}$ generate a tendency towards short-range chemical ordering in ZX10 by the formation $\mathrm{Zn}$-Ca co-clusters. This is experimentally validated in Fig. 12, which shows STEM-EDS elemental maps of ZX10 in the solutionized state. The non-random distribution of $\mathrm{Zn}$ (in red) and $\mathrm{Ca}$ (in green) atoms in the Mg matrix, with their tendency to jointly exist as clusters (greenish-yellow regions shown by white arrow heads), highlights the aforementioned chemical ordering effect. The forthcoming paragraph numerically estimates the local stress increment arising from the short-range ordering due to $\mathrm{Zn}-\mathrm{Ca}$ solutes.

Mechanistically, when a dislocation shears a solute cluster and thus destroys the local order, a local stress increment results that is directly proportional to the change in the interfacial energy related to the short-range order (SRO) per unit area, expressed as [56,57],

$\Delta \tau_{\mathrm{SRO}}=\frac{\gamma_{\mathrm{SRO}}}{|\mathbf{b}|} \approx \frac{f_{\text {cluster }}\left(E_{\mathrm{SRO}}-E_{\text {random }}\right)}{|\mathbf{b}|^{3}}$

where, $\gamma_{\mathrm{SRO}}$ is the energy change associated with destroying the locally ordered configuration per unit area, $E_{\mathrm{SRO}}$ is the short-range ordering energy, $E_{\text {random }}$ is the energy state of a random solid solution, and $f_{\text {cluster }}$ is the overall volume fraction of the co-clusters The latter can be assumed to be of a similar magnitude as the sum of the total atomic fraction of $\mathrm{Zn}$ and Ca solutes in $\mathrm{Mg}$, which amounts to around $0.7 \%$ (experimentally measured bulk composition: $\mathrm{Ca} \sim 0.18$ at.\%, $\mathrm{Zn} \sim 0.54$ at.\%). Furthermore, $|\mathbf{b}|$ corresponds to the magnitude of the Burgers vector of the active dislocation slip system, which is in this case $(0001)\langle 11 \overline{2} 0\rangle$ basal slip. For the sake of simplicity, we reasonably assume that $E_{\text {random }} \approx \Delta E_{\mathrm{Mg}-\mathrm{Mg}}$. This results from the lean-alloying approach where the number of $\mathrm{Mg}-\mathrm{Mg}$ bonds by far exceeds that of the $\mathrm{Mg}-\mathrm{Ca}$ and $\mathrm{Mg}-\mathrm{Zn}$ bonds in the matrix, such that the contribution of the $\mathrm{Mg}$-solute-element bonding to the total energy of the system can be neglected in the current scenario. Moreover, in the case of co-segregation of $\mathrm{Zn}-$ Ca solute clusters the ordering energy can be mathematically expressed as $E_{\mathrm{SRO}}=\Delta E_{\mathrm{Zn}-\mathrm{Ca}}$.

Inserting the values of $\Delta E_{\mathrm{Zn}-\mathrm{Ca}}$ and $\Delta E_{\mathrm{Mg}-\mathrm{Mg}}$ from Refs. $[26,54,58]$ into eqn. 3 gives the strength increment due to shortrange ordering, $\Delta \tau_{\mathrm{SRO}} \mathrm{ZnCa} \approx 27 \mathrm{MPa}$. Interestingly the theoretically calculated value of $\Delta \tau_{\mathrm{SRO}} \mathrm{ZnCa}$ is in very good agreement with the observed twin strengthening $\left(\Delta \tau_{\text {rel }}\right.$ twin $\sim 29 \mathrm{MPa}$ ) in ZX10 (see Fig. 11), thereby implying that such chemically driven local atomic rearrangement of solutes can play a significant role in terms of local-scale strengthening of zonal dislocation motion during twin nucleation. This results in relatively higher twinning stresses for the lean alloy ZX10 compared to pure Mg. In the forthcoming sections, the impact of short-range ordering on the spatial configuration and density of the defects will be elucidated, which leads to a significant improvement in the overall material strength-ductility response.

\subsection{Dislocation dynamics and slip characteristics}

Fig. 13 shows an analysis of the discrete stress-drop events, corresponding to independent dislocation bursts, detected during the mechanical tests on ZX10 compared to those performed on pure $\mathrm{Mg}$ under c-axis compression. Fig. 13a shows the number frequency of dislocation-burst events as a function of stress-drop magnitude (averaged over three stress-strain curves for each material), indicating a bimodal distribution for ZX10 (shown by the two peaks as highlighted by arrows in Fig. 13a). Moreover, the appearance of a shoulder between two peaks in the cumulative frequency distribution for the ZX10 alloy (which is significantly less pronounced for pure $\mathrm{Mg}$ ) relates to the overlap of two unimodal peaks, which 

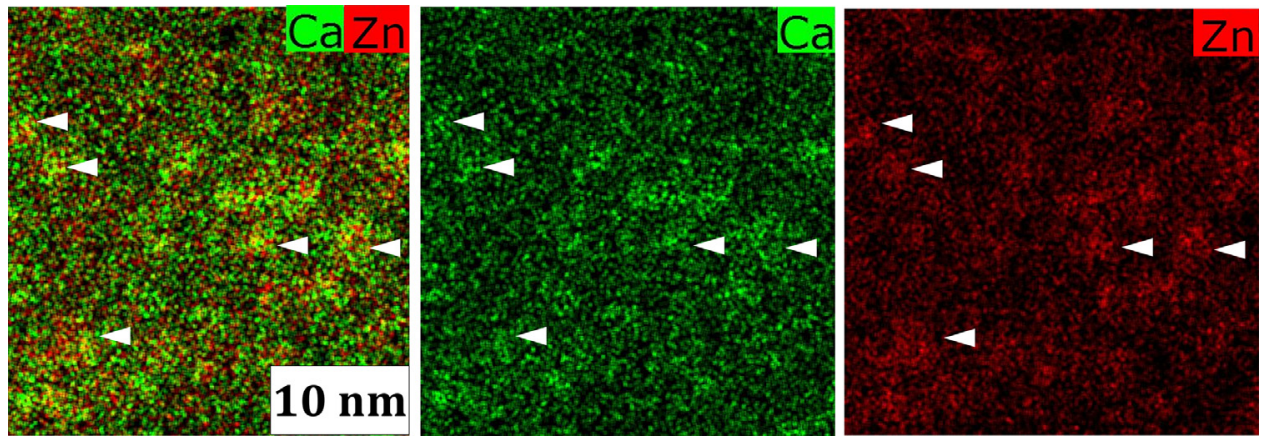

Fig. 12. STEM-EDS chemical maps of the $\mathrm{Zn}$ and Ca distributions in the bulk ZX10 alloy.
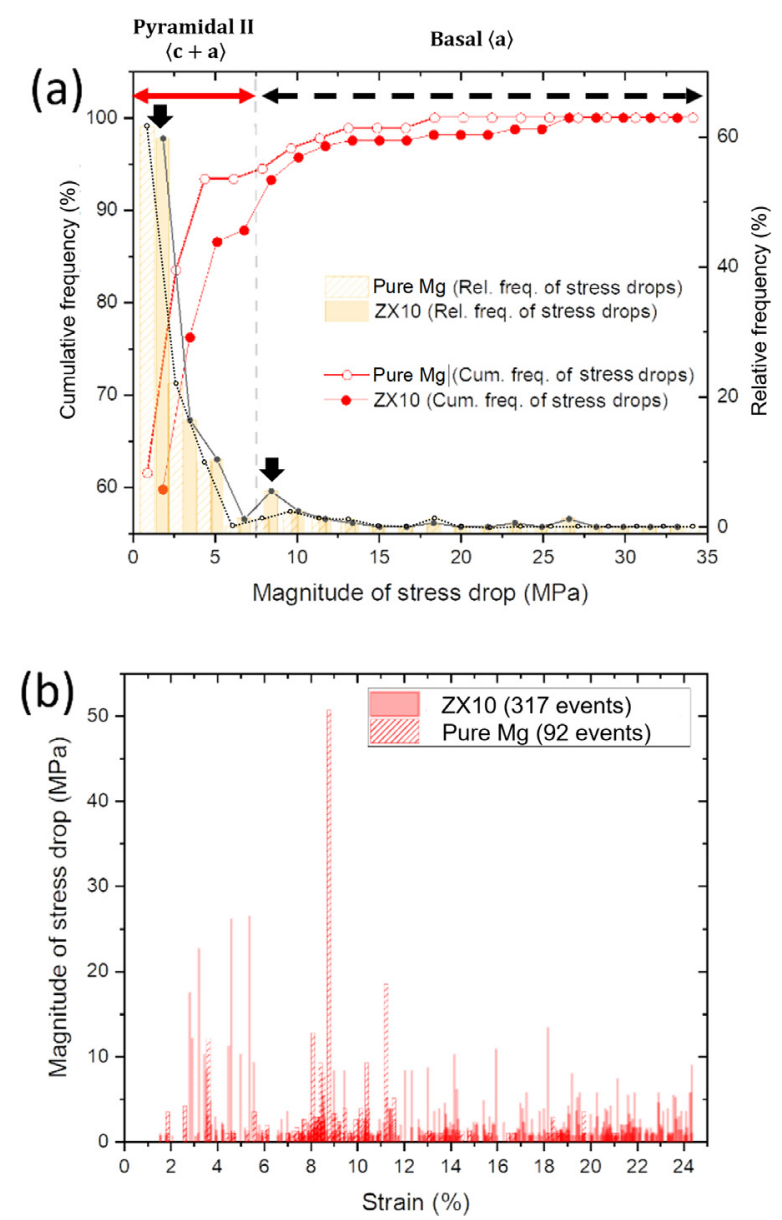

Fig. 13. Dislocation-burst event analysis under $c$-axis compression in terms of (a) cumulative and relative frequency of the number fraction of dislocation bursts as a function of stress-drop magnitude and (b) variation of stress-drop magnitude as a function of applied strain. The two regimes indicated by the solid red and dashed black double-arrows describe slip contributions from pyramidal II $\langle c+a\rangle$ slip and basal $\langle a\rangle$ slip, respectively.

confirms bimodality in the slip events for the ZX10 alloy. The second feature evident from the stress-drop distribution for ZX10 is the presence of a high-frequency peak with a low stress-drop magnitude and the simultaneous appearance of a low-frequency peak with a larger stress-drop magnitude. The aforementioned trends for ZX10 indicate simultaneous activation of basal $\langle a\rangle$ and pyramidal $\langle c+a\rangle$ slip inside the micropillars, and therefore also agree with the findings from t-EBSD and TEM characterization. TEM observations in Fig. 9 indicated a dense network of $\langle c+a\rangle$ dislocation segments that are primarily confined in-between periodically ar- ranged basal dislocations and stacking faults spanning across the pillar width. When considering the mean free paths for dislocation motion, the TEM results indicate significantly shorter distances traversed by the $\langle c+a\rangle$ dislocations but a higher dislocation density as opposed to basal dislocations, which have much lower spatial density but larger mean free paths. Since the plastic shear $(\delta)$ due to a dislocation-burst event (physically observed as a stress-drop event during deformation) is proportional to the distance $(x)$ traversed by each dislocation $(\delta \propto x)$ [59], the smaller mean free paths for $\langle c+a\rangle$ dislocations must statistically correspond to the peaks with lower stress-drop magnitude, whereas the stress peaks with higher magnitude and relatively lower number of occurrences comply with basal dislocations. Conversely, in the case of $c$-axis compression of pure $\mathrm{Mg}$, the unimodal distribution correlates with the activation of predominantly occurring basal $\langle a\rangle$ slip, as evident from massive basal sliding, which accounts for the drastic softening observed in the stress-strain response.

Fig. 13b shows the number and distribution of dislocation events (averaged over three independent load-displacement curves for each case) as a function of deformation strain for ZX10 and pure $\mathrm{Mg}$. The figure reveals much more dislocation-burst events for ZX10 (317 independent dislocation bursts) than for pure Mg (92 independent dislocation events). Furthermore, the dislocation-burst events in ZX10 are more homogeneously distributed with increasing strain and alternate between intervals of small stress fluctuations, corresponding to $\langle c+a\rangle$ slip, and singular instances of large stress drops, which are associated with basal $\langle a\rangle$ slip. The homogeneous activation of $\langle c+a\rangle$ pyramidal slip and basal $\langle a\rangle$ slip gives rise to more than five independent deformation modes, and thus to uniform strain hardening and plasticity as seen in the stressstrain curve under $c$-axis compression. This also explains the more gradual orientation change of the pillar under $c$-axis compression (see Fig. 6), where the slip-induced rotations associated with basal and non-basal slip have opposite signs, so that the net orientation change under deformation remains small.

On the other hand, the dislocation bursts in pure Mg are distributed heterogeneously with increasing strain without any definite pattern of stress drops unlike for ZX10 (see Fig. 13b). The smaller number of slip events and their non-uniform distribution typically highlight the anisotropy in the deformation of pure $\mathrm{Mg}$ and the lack of available deformation modes to accommodate the imposed plastic strain. This also explains the observed pillar instability for pure $\mathrm{Mg}$ at strains larger than $5 \%$.

On the basis of the aforementioned dislocation dynamics, the cyclic hardening and softening seen in the stress-strain curves of ZX10 under c-axis extension is explained as follows: Plasticity begins with predominant deformation twinning and concurrent prismatic $\langle a\rangle$ slip in the untwinned parent. As twinning exhausts, the reorientation of the twinned crystal aligns the $c$-axis along the compression direction. This leads to a rapid stress increment un- 
til pyramidal $\langle c+a\rangle$ slip systems activate, which by themselves offer five independent deformation modes, therefore ensuring a uniform strain-hardening behavior. Activation of non-basal slip also results in concurrent rotation of the $c$-axis of the twinned pillar away from the compression direction, whereby the crystallographically hard twin orientation (in terms of basal slip) becomes conducive to activate basal $\langle a\rangle$ slip. The activation of basal $\langle a\rangle$ slip results in strain softening events in the stress-strain curve (also corroborated by the appearance of basal shear steps in Fig. 3), until the slip-induced rotation again results in the alignment of the $c$-axis along the compression axis. This appears as second hardening regime in Fig. 1, where the non-basal slip systems activate and sustain the deformation until the pillar reorients again to facilitate basal slip. The alternate activation of basal and non-basal slip eventually results in a complex three-dimensional dislocation structure at higher strains, where grain fragmentation and dynamic recovery processes initiate and give rise to a polycrystalline microstructure in the ZX10 pillars (see Fig. 5). Such structural evolution is a direct indication of homogeneous strain accommodation across the pillar volume, which explains the simultaneous enhancement of strength and ductility in the ZX10 alloy. Contrarily, for pure $\mathrm{Mg}$ the two twin variants lead to a reorientation of the $c$-axes in the twinned parts by $\pm 15^{\circ}$ away from the compression axis (see Figs. 1 and 10b). This explains the relatively modest strain hardening after twinning, because basal $\langle a\rangle$ slip is still active (see Fig. 1) until the basal-slip deformation generates a gradual alignment of the $c$-axes in the twinned grains towards the compression axis. This marks the onset of a steep hardening regime, which in the absence of $\langle c+a\rangle$ slip eventually leads to crystallographic failure.

\subsection{Stacking-fault mediated simultaneous strength-ductility enhancement}

The current work shows that minute additions of $\mathrm{Zn}$ and Ca significantly modify the plasticity mechanisms in magnesium, giving rise to more homogeneous plasticity and enhanced strain hardening. We propose that the key mechanism driving such mechanical response in ZX10 is associated with the role of the alloying elements to intrinsically modify the stacking-fault energies. Experimental attempts to measure the equilibrium separation between partials of $\left(\delta_{0002}\right)$, which bind an $I_{2}$-type fault in ZX10, were performed on TEM images. The experiments reveal a spacing in the range of 20-50 $\mathrm{nm}$. The corresponding stacking-fault energy $\left(\gamma_{\mathrm{I}_{2}}\right)$ can be calculated as $[13,60,61]$

$\gamma_{I_{2}}=\frac{G\left|\mathbf{b}_{\text {Shockley }}\right|^{2}}{8 \pi \delta_{0002}} \frac{2-v}{1-v}\left(1-\frac{2 v}{2-v} \cos 2 \theta\right)$,

where $G$ is the shear modulus (17 GPa for pure $\mathrm{Mg}$ ), $v$ is the Poisson ratio ( 0.35 for $\mathrm{Mg}$ ), $\mathbf{b}_{\text {Shockley }}$ is the Burgers vector of a Shockley partial $\left(\frac{1}{3}\langle 10 \overline{1} 0\rangle\right)$, and $\theta$ is the angular separation between the Burgers vectors of the two partial dislocations, given as $60^{\circ}$ [61]. Inserting the numerical values we obtain $\gamma_{I_{2}}$ being on the order of $1.4-3.6 \mathrm{~mJ} / \mathrm{m}^{2}$ for $\mathrm{ZX} 10$, which is significantly lower than the values reported for pure $\mathrm{Mg}\left(30-80 \mathrm{~mJ} / \mathrm{m}^{2}\right)$ [62-65]. The values clearly illustrate an overall lowering of the stacking-fault energies in the presence of $\mathrm{Zn}$ and $\mathrm{Ca}$ solutes. While further work is needed to understand the detailed role of $\mathrm{Zn}$ and $\mathrm{Ca}$ solutes on the stabilization and lowering of the stacking-fault energy, the concept of Suzuki-segregation induced stacking-fault energy lowering stands as a plausible explanation.

In metallic alloys the stacking-fault energy results from both the contributions of elastic repulsion effects between partial dislocations and local chemistry fluctuations. The latter phenomenon is known as Suzuki effect [52,66]. Here, stacking faults can be thermodynamically treated as Gibbs dividing surface of finite width, whose free energy in a solid solution varies with the solute content, which generally differs from that of the neighbouring perfect crystal. The change in solute content in the stacking fault relates to the difference in chemical potential between the matrix and the stacking fault, which can either promote segregation of solutes to faults or vice-versa. The overall stacking-fault energy in an alloyed system $\left(\gamma_{\mathrm{SFE} \text { (alloyed) }}\right)$ is

$\gamma_{\mathrm{SFE}(\text { alloyed })}=\gamma_{\mathrm{SFE}(\text { pure })}+\Delta \gamma_{\mathrm{ch}}$,

where $\Delta \gamma_{\mathrm{ch}}=\sum_{i} \zeta_{i} \mathrm{~d} \mu_{i}$, and $\zeta_{i}$ and $\mathrm{d} \mu_{i}$ are the surface excess and chemical potential difference of solute $i$. When solute atoms segregate to the faulted area, $\gamma_{\operatorname{SFE} \text { (alloyed) }}$ is lowered and the equilibrium separation of partials increases, while depletion of solutes from the stacking fault results in a reverse effect.

A recent density functional theory (DFT) study performed on binary $\mathrm{Mg}$ alloys revealed that both $\mathrm{Zn}$ and Ca solute atoms show a propensity to segregate to stacking faults in $\mathrm{Mg}$, thereby contributing to a lowering of the stacking-fault energy [62,67-69]. In the present ternary $\mathrm{Mg}$ alloy, the presence of $\mathrm{Zn}$ and $\mathrm{Ca}$ solutes in the form of chemically ordered clusters in the Mg matrix is expected to further amplify the chemical interaction and resultant segregation to planar crystal defects, i.e. stacking faults. A second contributing factor for $\mathrm{Zn}$ and $\mathrm{Ca}$ co-segregation to the faulted surface is the minimization of elastic strain in the vicinity of the fault $[26,61]$. Our assumption of a reduced stacking-fault energy in the alloyed state due to stabilization by solute segregation is further corroborated by the TEM images of the undeformed regions in the solution-treated ZX10 alloy, where densely populated pre-existing basal stacking faults are visible (see Fig. 14), unlike in the case of pure Mg [70].

The implications of lowered stacking-fault energies on the deformation response in magnesium are significant. In particular, lower SFE is known to facilitate the nucleation and mobility of pyramidal dislocations, which are often difficult to activate. In principle, two types of intrinsic basal stacking faults are energetically feasible in $\mathrm{Mg}$ and its alloys [71]: (i) the previously mentioned in-plane $I_{2}$-type deformation fault and (ii) the out-of-plane $I_{1}$-type growth fault, which is bound by Frank partials (FPs) and defined

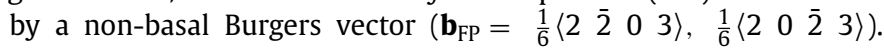
A decrease in $\gamma_{I_{2}}$ manifests as reduced in-plane mobility of the basal-dislocation structure and restricts the cross-slip of basal $\langle a\rangle$ dislocations. This invariably promotes basal-slip hardening and increases the chances of non-basal dislocation activation. On the other hand, a reduction in $\gamma_{I_{1}}$ would promote and stabilize dislocation dissociation into Frank partials, which would very likely facilitate the activation of pyramidal $\langle c+a\rangle$ sources. The overall effect of the lowering of the stacking-fault energy by $\mathrm{Zn}$ and $\mathrm{Ca}$ addition can therefore explain the observed enhanced $\langle c+a\rangle$ activity in the ZX10 alloy. The experimentally-measured critical resolved shearstress (CRSS) values to activate pyramidal $\langle c+a\rangle$ slip are for ZX10 $(123 \pm 5 \mathrm{MPa})$ significantly lower than that for pure $\mathrm{Mg}(189 \pm 6.5$ $\mathrm{MPa}$ ), which indicates significantly easier pyramidal slip nucleation in the ZX10 alloy.

In light of the above mechanisms, the operative slip systems, the strain hardening, and the strengthening behavior are all mediated by the stacking-fault energy in ZX10. Fig. 15a shows a schematic image of the defect structure in ZX10 as reconstructed from the TEM micrographs shown in Figs. 15b and 15c. Such appearance of $I_{1}$-type fault loops as observed in Fig. 15b has previously been reported for $\mathrm{Mg}$ alloys [72,73]. While the presence of $I_{1}$ fault loops facilitates the nucleation of $\langle c+a\rangle$ dislocations that promote ductility (see Fig. 15b), the $I_{2}$ stacking faults that traverse the pillar width also simultaneously act as barriers to $\langle c+a\rangle$ dislocation motion, giving rise to strong strain hardening on the pyramidal planes. Furthermore, the interaction of $\langle c+a\rangle$ screw segments 


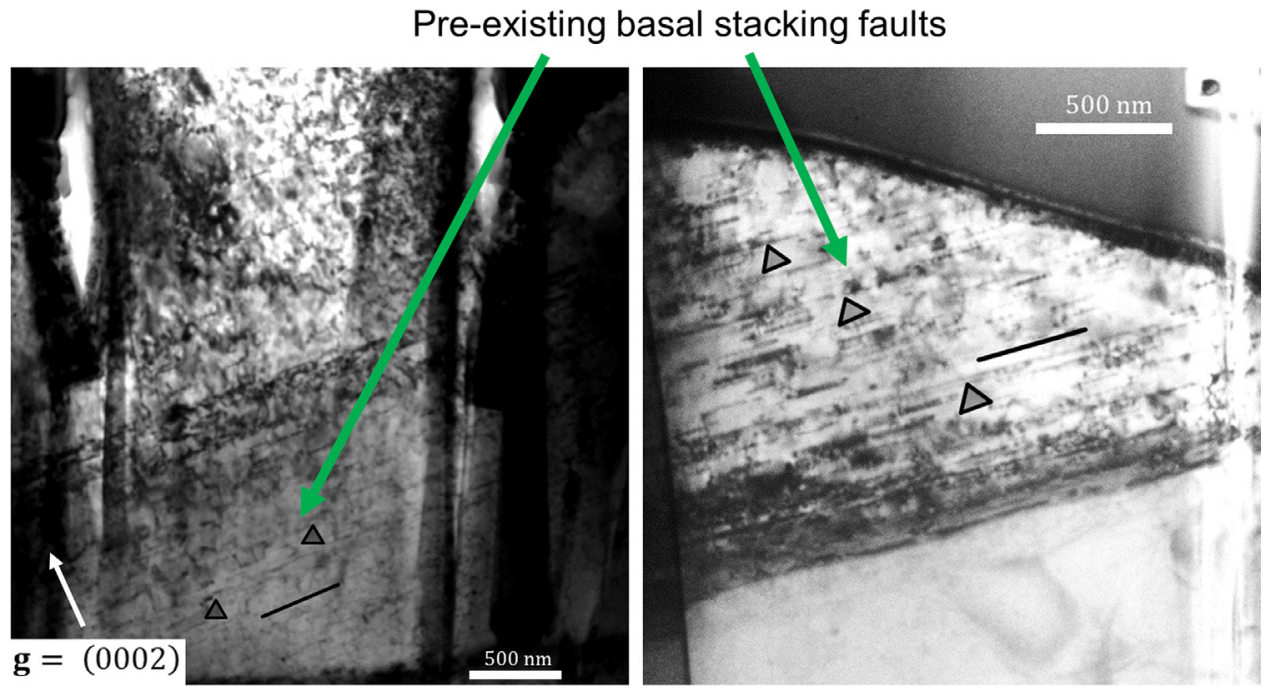

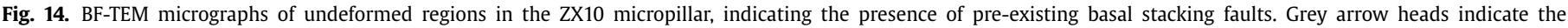
narrow streaks corresponding to basal stacking faults and black line represents the basal $\langle a\rangle$ slip.

(a)

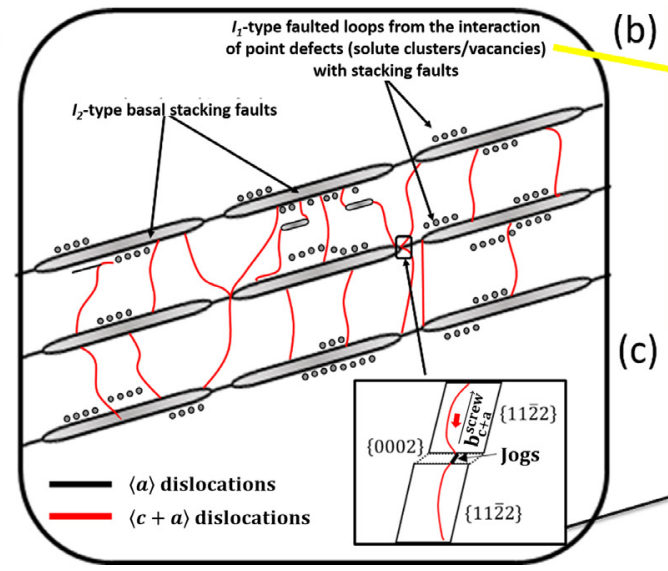

(b)

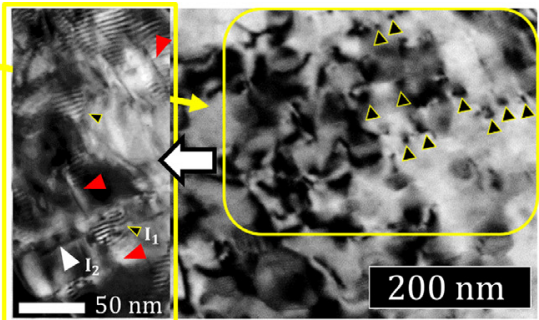

(c)

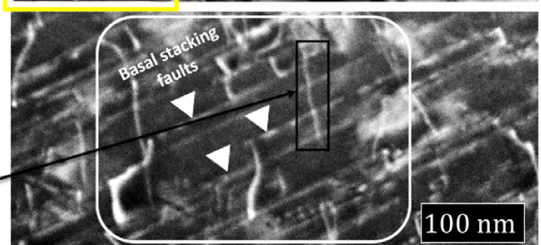

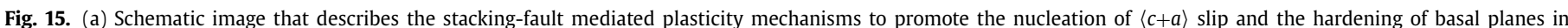

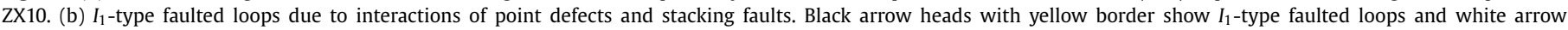

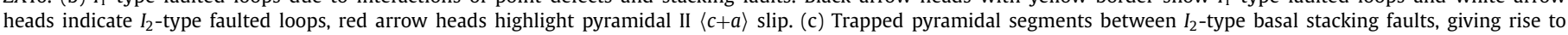
dislocation jogs in the basal planes.

with the basal planes generates dislocation jogs in the basal plane (see Figs. 15a and 15c), which further restrict the mobility of basal dislocations in addition to the aforementioned role played by the $I_{2}$ stacking faults. Both contributions promote the hardening of basal dislocations and thus explain the uniform strengthening regime observed during the deformation of ZX10 under c-axis compression. However, the additional activated slip systems provide enhanced ductility, despite these strengthening obstacles.

\section{Conclusion}

We studied the plasticity and strengthening mechanisms in single-crystalline solid-solution lean $\mathrm{Mg}-\mathrm{Zn}-\mathrm{Ca}$ alloys and, for comparison, in pure $\mathrm{Mg}$ via micromechanical testing and correlative electron microscopy techniques. We can draw the following key conclusions:

1 Single-crystalline micro-pillars of ZX10 and pure Mg were strained under c-axis compression and c-axis extension. In the case of $c$-axis extension ZX10 reveals a strengthening increment by a factor of 2 compared to pure $\mathrm{Mg}$, with simultaneous ductility enhancement. Under $c$-axis compression, the strengthen- ing increment for ZX10 alloys is a factor of 2.5 higher than for pure $\mathrm{Mg}$, with a simultaneous ductility enhancement of $~ 33 \%$.

2 Deforming ZX10 under $c$-axis extension results in twinningmediated plasticity at low strains, highlighted by $\{10 \overline{1} 2\}$ tension twins. At higher strains various nano-scale twins appear in the form of secondary $\{10 \overline{1} 2\}-\{10 \overline{1} 2\}$ twins plus low Schmidfactor $\{10 \overline{1} 2\}$ tension twin variants and $\{10 \overline{1} 1\}$ compression twins at regions of local stress pile-up along the twin-parent interface. The twinned grains undergo simultaneous slip along both basal and pyramidal planes, giving rise to homogeneous deformation. A further strain increment results in grain fragmentation and dynamic recovery in the regions of high stress concentration. In contrast, pure $\mathrm{Mg}$ reveals plasticity that is primarily driven by tension twinning and basal slip.

3 Twin-nucleation stress values under $c$-axis extension indicate twin strengthening by local chemical ordering, which is in the solid-solution ZX10 alloy $29 \mathrm{MPa}$ higher than in pure Mg.

4 Under $c$-axis compression ZX10 deforms homogeneously along the pillar volume via $\langle c+a\rangle$ pyramidal slip and basal $\langle a\rangle$ slip. In contrast, pure Mg shows severe basal sliding, which results in an early onset of plastic instability in the pillar. 
5 Addition of $\mathrm{Zn}$ and Ca lowers the overall stacking-fault energy of pure $\mathrm{Mg}$ by a factor of $\sim 20$. The resultant effect leads to lower activation stress (CRSS values) and easier pyramidal $\langle c+a\rangle$ dislocation mobility in the ZX10 alloy, together with a restriction of basal-slip mobility, giving rise to strong basal hardening. The presence of stacking faults additionally influences the spatial arrangement of dislocations. $I_{1}$ faults promote nucleation of pyramidal dislocations, and $I_{2}$ faults act as active barriers to incoming pyramidal dislocations, giving rise to a synergistic plasticity and strengthening enhancement.

\section{Declaration of Competing Interest}

The authors declare that they have no known competing financial interests or personal relationships that could have appeared to influence the work reported in this paper.

\section{Acknowledgements}

Support by the ETH+ initiative within the framework of SynMatLab (Laboratory for Multiscale Materials Synthesis and HandsOn Education) and by the Swiss National Science Foundation (Grant No. 200021-172934) is gratefully acknowledged. The authors also thank the Scientific Center for Optical and Electron Microscopy (ScopeM), ETH Zurich, for access to its facilities.

\section{Supplementary materials}

Supplementary material associated with this article can be found, in the online version, at doi:10.1016/j.actamat.2021.116877.

\section{References}

[1] B.L. Mordike, T. Ebert, Magnesium: Properties - applications - potential, Mater. Sci. Eng. A 302 (1) (2001) 37-45.

[2] J. Hirsch, T. Al-Samman, Superior light metals by texture engineering: Optimized aluminum and magnesium alloys for automotive applications, Acta Materialia 61 (3) (2013) 818-843.

[3] I. Basu, T. Al-Samman, Triggering rare earth texture modification in magnesium alloys by addition of zinc and zirconium, Acta Materialia 67 (2014) 116-133.

[4] M.M. Avedesian, H. Baker, ASM specialty handbook: magnesium and magnesium alloys, ASM international, 1999.

[5] T.M. Pollock, Weight Loss with Magnesium Alloys, Science 328 (5981) (2010) 986-987.

[6] E. Aghion, B. Bronfin, Magnesium alloys development towards the 21st century, Materials Science Forum, Trans. Tech. Publ. (2000) 19-30.

[7] Z. Wu, W.A. Curtin, The origins of high hardening and low ductility in magnesium, Nature 526 (2015) 62.

[8] M.H. Yoo, Slip, twinning, and fracture in hexagonal close-packed metals, Metal. Trans. A 12 (3) (1981) 409-418.

[9] M.H. Yoo, J.K. Lee, Deformation twinning in h.c.p. metals and alloys, Philos. Mag. A 63 (5) (1991) 987-1000.

[10] I. Basu, T. Al-Samman, Twin recrystallization mechanisms in magnesium-rare earth alloys, Acta Materialia 96 (2015) 111-132.

[11] I. Basu, T. Al-Samman, Competitive twinning behavior in magnesium and its impact on recrystallization and texture formation, Mater. Sci. Eng. A 707 (2017) 232-244.

[12] Z. Wu, R. Ahmad, B. Yin, S. Sandlöbes, W.A. Curtin, Mechanistic origin and prediction of enhanced ductility in magnesium alloys, Science 359 (6374) (2018) 447.

[13] S. Sandlöbes, M. Friák, S. Zaefferer, A. Dick, S. Yi, D. Letzig, Z. Pei, L.F. Zhu, J. Neugebauer, D. Raabe, The relation between ductility and stacking fault energies in Mg and Mg-Y alloys, Acta Materialia 60 (6) (2012) 3011-3021.

[14] S. Sandlöbes, S. Zaefferer, I. Schestakow, S. Yi, R. Gonzalez-Martinez, On the role of non-basal deformation mechanisms for the ductility of $\mathrm{Mg}$ and $\mathrm{Mg}-\mathrm{Y}$ alloys, Acta Materialia 59 (2) (2011) 429-439.

[15] I. Basu, T. Al-Samman, G. Gottstein, Shear band-related recrystallization and grain growth in two rolled magnesium-rare earth alloys, Mater. Sci. Eng. A 579 (2013) 50-56

[16] R. Ahmad, Z. Wu, S. Groh, W.A. Curtin, Pyramidal II to basal transformation of $\langle c+$ a $\rangle$ edge dislocations in Mg-Y alloys, Scripta Materialia 155 (2018) 114-118.

[17] R. Ahmad, B. Yin, Z. Wu, W.A. Curtin, Designing high ductility in magnesium alloys, Acta Materialia 172 (2019) 161-184.

[18] S. Sandlöbes, M. Friák, S. Korte-Kerzel, Z. Pei, J. Neugebauer, D. Raabe, A rare-earth free magnesium alloy with improved intrinsic ductility, Sci. Rep.7 (1) (2017) 10458.
[19] M. Cihova, R. Schäublin, L.B. Hauser, S.S.A. Gerstl, C. Simson, P.J. Uggowitzer, J.F. Löffler, Rational design of a lean magnesium-based alloy with high age-hardening response, Acta Materialia 158 (2018) 214-229.

[20] T.T.T. Trang, J.H. Zhang, J.H. Kim, A. Zargaran, J.H. Hwang, B.C. Suh, N.J. Kim, Designing a magnesium alloy with high strength and high formability, Nat. Commun.9 (1) (2018) 2522

[21] Z.R. Zeng, Y.M. Zhu, S.W. Xu, M.Z. Bian, C.H.J. Davies, N. Birbilis, J.F. Nie, Texture evolution during static recrystallization of cold-rolled magnesium alloys, Acta Materialia 105 (2016) 479-494.

[22] F. Witte, N. Hort, C. Vogt, S. Cohen, K.U. Kainer, R. Willumeit, F. Feyerabend, Degradable biomaterials based on magnesium corrosion, Curr. Opin. Solid State Mater. Sci.12 (5) (2008) 63-72.

[23] J. Hofstetter, S. Rüedi, I. Baumgartner, H. Kilian, B. Mingler, E. Povoden-Karadeniz, S. Pogatscher, P.J. Uggowitzer, J.F. Löffler, Processing and microstructure-property relations of high-strength low-alloy (HSLA) Mg-Zn-Ca alloys, Acta Materialia 98 (2015) 423-432.

[24] J. Hofstetter, M. Becker, E. Martinelli, A.M. Weinberg, B. Mingler, H. Kilian, S. Pogatscher, P.J. Uggowitzer, J.F. Löffler, High-Strength Low-Alloy (HSLA) Mg-Zn-Ca Alloys with Excellent Biodegradation Performance, JOM 66 (4) (2014) 566-572.

[25] J.D. Robson, Effect of rare-earth additions on the texture of wrought magnesium alloys: the role of grain boundary segregation, Metallurg. Mater. Trans. A 45 (8) (2014) 3205-3212.

[26] I. Basu, K.G. Pradeep, C. Mießen, L.A. Barrales-Mora, T. Al-Samman, The role of atomic scale segregation in designing highly ductile magnesium alloys, Acta Materialia 116 (2016) 77-94.

[27] C. Drouven, I. Basu, T. Al-Samman, S. Korte-Kerzel, Twinning effects in deformed and annealed magnesium-neodymium alloys, Mater. Sci. Eng. A 647 (2015) 91-104

[28] I.-H. Jung, M. Sanjari, J. Kim, S. Yue, Role of RE in the deformation and recrystallization of $\mathrm{Mg}$ alloy and a new alloy design concept for $\mathrm{Mg}-\mathrm{RE}$ alloys, Scripta Materialia 102 (2015) 1-6.

[29] D. Griffiths, Explaining texture weakening and improved formability in magnesium rare earth alloys, Mater. Sci. Tech.31 (1) (2015) 10-24.

[30] J. Bohlen, M.R. Nürnberg, J.W. Senn, D. Letzig, S.R. Agnew, The texture and anisotropy of magnesium-zinc-rare earth alloy sheets, Acta Materialia 55 (6) (2007) 2101-2112.

[31] K. Hantzsche, J. Bohlen, J. Wendt, K.U. Kainer, S.B. Yi, D. Letzig, Effect of rare earth additions on microstructure and texture development of magnesium alloy sheets, Scripta Materialia 63 (7) (2010) 725-730.

[32] T. Al-Samman, X. Li, Sheet texture modification in magnesium-based alloys by selective rare earth alloying, Mater. Sci. Eng. A 528 (10) (2011) 3809-3822.

[33] J.-F. Nie, Precipitation and Hardening in Magnesium Alloys, Metallurg. Mater. Trans. A 43 (11) (2012) 3891-3939.

[34] J.F. Nie, B.C. Muddle, Precipitation hardening of $\mathrm{Mg}-\mathrm{Ca}(-\mathrm{Zn})$ alloys, Scripta Materialia 37 (10) (1997) 1475-1481.

[35] F. Bachmann, R. Hielscher, H. Schaeben, Texture Analysis with MTEX - Free and Open Source Software Toolbox, Solid State Phenomena 160 (2010) 63-68.

[36] S.I. Wright, M.M. Nowell, S.P. Lindeman, P.P. Camus, M. De Graef, M.A. Jackson, Introduction and comparison of new EBSD post-processing methodologies, Ultramicroscopy 159 (2015) 81-94.

[37] R. Schäublin, P. Stadelmann, A method for simulating electron microscope dislocation images, Mater. Sci. Eng. A 164 (1) (1993) 373-378.

[38] L.J. Slutsky, C.W. Garland, Elastic Constants of Magnesium from 4.2K to 300K, Phys. Rev.107 (4) (1957) 972-976.

[39] K.E. Prasad, K. Rajesh, U. Ramamurty, Micropillar and macropillar compression responses of magnesium single crystals oriented for single slip or extension twinning, Acta Materialia 65 (2014) 316-325.

[40] Q. Yu, L. Qi, K. Chen, R.K. Mishra, J. Li, A.M. Minor, The Nanostructured Origin of Deformation Twinning, Nano Letters 12 (2) (2012) 887-892.

[41] I. Basu, V. Ocelík, J.T.M. De Hosson, Measurement of spatial stress gradients near grain boundaries, Scripta Materialia 136 (2017) 11-14.

[42] M.R. Barnett, Twinning and the ductility of magnesium alloys: Part I: "Tension" twins, Mater. Sci. Eng. A 464 (1) (2007) 1-7.

[43] W.A.T. Clark, R.H. Wagoner, Z.Y. Shen, T.C. Lee, I.M. Robertson, H.K. Birnbaum, On the criteria for slip transmission across interfaces in polycrystals, Scripta Metallurgica et Materialia 26 (2) (1992) 203-206.

[44] Z. Shen, R.H. Wagoner, W.A.T. Clark, Dislocation and grain boundary interactions in metals, Acta Metallurgica 36 (12) (1988) 3231-3242.

[45] I. Basu, J.T.M. De Hosson, High Entropy Alloys: Ready to Set Sail? Metals 10 (2) (2020) 194.

[46] Y. Liu, N. Li, M. Arul Kumar, S. Pathak, J. Wang, R.J. McCabe, N.A. Mara, C.N. Tomé, Experimentally quantifying critical stresses associated with basal slip and twinning in magnesium using micropillars, Acta Materialia 135 (2017) 411-421.

[47] J. Wang, J.P. Hirth, C.N. Tomé, (1-012) Twinning nucleation mechanisms in hexagonal-close-packed crystals, Acta Materialia 57 (18) (2009) 5521-5530.

[48] X. Liao, J. Wang, J. Nie, Y. Jiang, P. Wu, Deformation twinning in hexagonal materials, MRS Bulletin 41 (4) (2016) 314-319.

[49] Y.P. Cai, G.J. Wang, Y.J. Ma, Z.H. Cao, X.K. Meng, High hardness dual-phase high entropy alloy thin films produced by interface alloying, Scripta Materialia 162 (2019) 281-285.

[50] J. Wang, I.J. Beyerlein, C.N. Tomé, An atomic and probabilistic perspective on twin nucleation in Mg, Scripta Materialia 63 (7) (2010) 741-746.

[51] R.L. Fleischer, Substitutional solution hardening, Acta Metallurgica 11 (3) (1963) 203-209. 
[52] H. Suzuki, Segregation of Solute Atoms to Stacking Faults, J. Phys. Soc. Jpn. 17 (2) (1962) 322-325.

[53] R. Labusch, A Statistical Theory of Solid Solution Hardening, Physica Status Solidi (B) 41 (2) (1970) 659-669.

[54] A. Takeuchi, A. Inoue, Classification of Bulk Metallic Glasses by Atomic Size Difference, Heat of Mixing and Period of Constituent Elements and Its Application to Characterization of the Main Alloying Element, Mater. Trans.46 (12) (2005) 2817-2829.

[55] Y.F. Zhao, D.Y. Lin, X.H. Chen, Z.K. Liu, X.D. Hui, Sluggish mobility and strong icosahedral ordering in Mg-Zn-Ca liquid and glassy alloys, Acta Materialia 67 (2014) 266-277.

[56] J.C. Fisher, On the strength of solid solution alloys, Acta Metallurgica 2 (1) (1954) 9-10.

[57] M.J. Starink, S.C. Wang, The thermodynamics of and strengthening due to coclusters: General theory and application to the case of Al-Cu-Mg alloys, Acta Materialia 57 (8) (2009) 2376-2389.

[58] Y.-R. Luo, Comprehensive handbook of chemical bond energies, CRC press, 2007.

[59] I. Basu, V. Ocelík, J.T.M. De Hosson, Size dependent plasticity and damage response in multiphase body centered cubic high entropy alloys, Acta Materialia 150 (2018) 104-116.

[60] W.T. Read, Dislocations in crystals, McGraw-Hill, 1953.

[61] Y.M. Zhu, A.J. Morton, M. Weyland, J.F. Nie, Characterization of planar features in Mg-Y-Zn alloys, Acta Materialia 58 (2) (2010) 464-475.

[62] N. Chetty, M. Weinert, Stacking faults in magnesium, Phys. Rev. B - Condens. Matter Mater. Phys.56 (17) (1997) 10844-10851.

[63] A. Couret, D. Caillard, An in situ study of prismatic glide in magnesium-II. Microscopic activation parameters, Acta Metallurgica 33 (8) (1985) 1455-1462.
[64] D.H. Sastry, Y.V.R.K. Prasad, K.I. Vasu, On the stacking fault energies of some close-packed hexagonal metals, Scripta Metallurgica 3 (12) (1969) 927-929.

[65] A.E. Smith, Surface, interface and stacking fault energies of magnesium from first principles calculations, Surf. Sci.601 (24) (2007) 5762-5765.

[66] J.P. Hirth, Thermodynamics of stacking faults, Metallurg. Trans.1 (9) (1970) 2367.

[67] M. Muzyk, Z. Pakiela, K.J. Kurzydlowski, Generalized stacking fault energy in magnesium alloys: Density functional theory calculations, Scripta Materialia 66 (5) (2012) 219-222.

[68] W.Y. Wang, S.L. Shang, Y. Wang, Z.-G. Mei, K.A. Darling, L.J. Kecskes, S.N. Mathaudhu, X.D. Hui, Z.-K. Liu, Effects of Alloying Elements on Stacking Fault Energies and Electronic Structures of Binary Mg Alloys: A First-Principles Study, Mater. Res. Lett.2 (1) (2014) 29-36.

[69] Y.F. Wu, S. Li, Z.G. Ding, W. Liu, Y.H. Zhao, Y.T. Zhu, Effect of charge redistribution factor on stacking-fault energies of Mg-based binary alloys, Scripta Materialia 112 (2016) 101-105.

[70] Z. Pei, L.F. Zhu, M. Friák, S. Sandlöbes, J. von Pezold, H.W. Sheng, C.P. Race, S. Zaefferer, B. Svendsen, D. Raabe, J. Neugebauer, Ab initioand atomistic study of generalized stacking fault energies in $\mathrm{Mg}$ and $\mathrm{Mg}-\mathrm{Y}$ alloys, N. J. Phys.15 (4) (2013) 043020.

[71] P.G. Partridge, The crystallography and deformation modes of hexagonal closepacked metals, Metallurg. Rev.12 (1) (1967) 169-194.

[72] S. Agnew, J. Horton, M. Yoo, Transmission electron microscopy investigation of $\langle\mathrm{C}+\mathrm{a}>$ dislocations in $\mathrm{Mg}$ and $\alpha$-solid solution Mg-Li alloys, Metallurg. Mater Trans. A 33 (3) (2002) 851-858.

[73] P.M. Anderson, J.P. Hirth, J. Lothe, Theory of dislocations, Cambridge University Press, 2017. 